ALEXANDER SOMEK

\title{
Die Macht der juristischen Expertise Rechtliches Wissen als Steuerungsmedium
}

\section{Der Preis und der Wert des Rechts}

In Gesellschaften, die, weil sie niemand durchschaut, als "komplex" bezeichnet werden, wird das, was man als "geltendes Recht" bezeichnet, von juristischen Experten gewusst. ${ }^{1}$ Insofern deren Expertise eine Dienstoder Arbeitsleistung darstellt, wird sie entgelttich abgegeben. Juristische Expertise gibt es in der Regel nicht kostenlos. Das Erstellen von Erledigungen, Eingaben, Anbringen, Klagen, Gegenklagen, Berufungen oder Gutachten ist, wie Oliver Wendell Holmes zu Recht festgestellt hat, zunächst einmal ein Geschäft. ${ }^{2}$

Aber es ist ein Geschäft der besonderen Art. Die Zufriedenheit der Konsumenten ist, jedenfalls was behördliche Erledigungen angeht, keine Garantie ihres Wertes. Von Wert ist rechtliches Wissen nur, wenn es das geltende Recht darstellt. Eine vertrauenswürdige juristische Expertise hat mit dem Anspruch auf Rechtserkenntnis aufzutreten. Sie hat die richtige Antwort auf eine Rechtsfrage zu enthalten. ${ }^{3}$ Sie hat sogar selbst zu indizieren, dass sie weiß, weshalb dem so ist. Sie muss Selbststeuerungsfähigkeit indizieren. Der Experte zeigt an, dass er weiß und warum er weiß, was rechtens ist. Deswegen weiß er sich auch über den Widerstreit von politischen und wirtschaftlichen Interessen erhaben.

Das ist die ideale Version der juristischen Expertise. Was in modernen Gesellschaften von Experten als Recht gewusst wird, tritt in dieser Form auf. Die Expertise signalisiert Wissen. Sie signalisiert sogar praktisches Wissen. Mit Wissen dieser Art kann man etwas anfangen. ${ }^{4}$ Und

\footnotetext{
1 Für eine erste Annäherung an diese Thematik siehe ALEXANDER SOMEK, Juristische Expertise, in: KENT D. LERCH (Hrsg.), Recht verstehen. Verständlichkeit, Missverständlichkeit und Unverständlichkeit von Recht. Berlin 2004, 413-428.

2 Siehe Oliver Wendell Holmes, The Path of the Law, reprinted in: Harvard Law Review 110 (1997), 991-1009 (Erstveröffentlichung 1897).

${ }^{3}$ Siehe ROLAND DWORKIN, A Matter of Principle. Cambridge (Mass.) 1985.

${ }^{4}$ Siehe NiCO STEHR, Die Zerbrechlichkeit moderner Gesellschaften. Die Stagnation der Macht und die Chance des Individuums. Weilerswist 2000, 81-83.
} 
sie signalisiert, dass bei Bedarf die Gründe für dieses Wissens dargelegt werden könnten.

Das Signal der Begründung entlastet von ihrem Vollzug. Genau das wird durch die Verwendung der dem Laien unbequemen und undurchsichtigen juristischen Fachsprache geleistet. Die Semantik und Syntax der rechtswissenschaftlichen Expertise, jene seltsame Mischung von altehrwürdiger Fachterminologie und Respekt gebietendem Kanzleistil, indiziert, dass die Argumentierende über bewährte oder begründbare Prämissen verfügt und damit in der Lage wäre, nötigenfalls detailliert anzugeben, worin der Wert einer fachlichen Auskunft oder einer bindenden Entscheidung besteht.

Mit der juristischen Fachsprache ist dem rechtlichen Wissen also ein Ausdrucksmedium zugeordnet. Sein Gebrauch zeigt eine Einheit von praktischem Wissen und seiner Begründung an. Diese Einheit hat normativen Charakter. Verwertbar darf rechtliches Wissen nur dann sein, wenn es wissenschaftlich stichhaltig ist. Konzediert ist dabei, dass die wissenschaftliche Fundierung, um effiziente Verwertung zu gewährleisten, in anderen als den relevanten Handlungskontexten zu reflektieren ist. Anders wäre praktisches rechtliches Wissen nicht möglich. Es muss, um existieren zu können, von der Bürde entlastet sein, jederzeit vollständig und hinreichend begründet zu sein (siehe Tab. 1).

\begin{tabular}{c|c|c} 
& Nichthoheitlich & Hoheitlich \\
\hline $\begin{array}{c}\text { Institutionelle } \\
\text { Form }\end{array}$ & $\begin{array}{c}\text { Rechtsauskunft } \\
\text { (Rechtsbeistand) }\end{array}$ & Staatlicher Rechtsakt \\
\hline Preis & Tarif (Honorar) & Gehalt (Verfahrenskosten) \\
\hline Wert & & Juristische Expertise \\
\hline Deckung & & Juristische Selbstreflexion
\end{tabular}

Tab. 1: Die ideale Version der juristischen Expertise 
Vermöge dieses Anspruchs auf vernünftige Einheit in der Differenz von Operation und Legitimierung fungieren die theoretischen Prämissen der juristischen Expertise gleichsam wie ein Goldstandard. Und da zum Zwecke der praktischen Problemlösung die Expertise von der Erörterung grundsätzlicher Fragen zu entlasten ist, kann man sagen, dass die Rechtssprache als Zeichen der Validität juristischer Argumente deren soziale Akzeptanz auf eine Art und Weise sichert, die von Soziologen für symbolisch generalisierte Interaktionsmedien herausgearbeitet worden ist. ${ }^{5}$ Ich werde diese im Weiteren einfach als "Steuerungsmedien" bezeichnen, weil mich die sozialen Bedingungen interessieren, unter denen die juristische Expertise Autorität hat.

Im Rahmen der nun folgenden Rekonstruktion eines im einzelnen recht unterschiedlich vorgetragenen Theorieansatzes ${ }^{6}$ lässt sich zeigen, dass sich in der juristischen Expertise das gesellschaftlich verbindliche Medium ausgebildet hat, vermöge dessen gewusst wird, was das geltende Recht ist. ${ }^{7}$

${ }^{5}$ Siehe als exemplarische Stellungnahmen nur TALCOTT PARSONS, On the Concept of Political Power, in: Ders., Sociological Theory and Modern Society. New York 1967, 297254; DeRS., Zur Theorie der sozialen Interaktionsmedien. Hrsg. von STEFAN JENSEN. Opladen 1980, sowie aus jüngerer Zeit BERNHARD GIESEN, Die Entdinglichung des Sozialen. Eine evolutionstheoretische Perspektive auf die Postmoderne. Frankfurt am Main 1991, 223-243.

${ }^{6}$ Siehe zu einem Vergleich zwischen Parsons und Habermas bei WERNER GEPHART, Gesellschaftstheorie und Recht. Das Recht im soziologischen Diskurs der Moderne. Frankfurt am Main 1993, 164-167.

7 In der soziologischen Diskussion wird zưweilen dem Recht selbst die Qualität eines Steuerungsmediums zugesprochen. So lässt sich beispielsweise Münch von Parsons Idee, dass Medien als Symbole "the real thing" vertreten, dazu verleiten, subjektive Rechte und generelle Rechtsregeln als Zeichen für auszuführende Handlungen zu betrachten. Siehe RICHARD MüNCH, Recht als Medium der Kommunikation, in: Zeitschrift für Rechtssoziologie 13 (1992), 67-87, hier: 72-73, 79 und zur Symbolfunktion der Medien TALCOTT PARSONS, On the Concept of Influence, in: DERS., Sociological Theory and Modern Society (Fn. 5), 355-383, hier: 358, 367. Da sonach das Rechtsmedium aus der Perspektive subjektiver Rechte seine eigene Durchsetzung bezeichnet, bemisst sich der Wert eines Rechts nach Münch an der "Zahl der Handlungen", die durch den Gebrauch des Mediums bei anderen ausgelöst werden können (ebd., 79). Da Münch diese Wertbestimmung unabhängig davon ansetzt, ob ein Rechtsanspruch zu Recht oder zu Unrecht erhoben wird, bemisst sich der Wert eines Rechtes bloß danach, ob seine Durchsetzung den Wünschen des Berechtigten entspricht. Diese ohnehin reichlich abwegige Idee führt zu widersprüchlichen Konsequenzen, da durch sie nahe gelegt wird, dass der Wert eines Rechtes gleich Null sein müsste, wenn es sich im Zustand vollständiger Befriedigung befände, weil es dann „nichts zu kommunizieren“ (also zu klagen) gäbe.

Davon abgesehen ist es aber auch nicht zielführend, das Recht von der Art und Weise, wie es gesellschaftlich verbindlich gewusst wird, nicht zu unterscheiden. Alle Steuerungsmedien sind in einem rechtlich fundierten institutionellen Code verankert. Es ist daher nicht plausibel, dieser Verankerung selbst mediale Qualitäten zu unterschieben. Siehe GEPHART, Gesellschaftstheorie und Recht (Fn. 6), 247-251. Andernfalls riskiert man, dass die Konturen verschwimmen, vor allem was die genauere Bestimmung des Verhältnisses von Recht und Macht betrifft. Letzteres ist deutlich bei JÜRGEN 
Ich beabsichtige aber nicht bloß, den theoretischen Analyserahmen zu skizzieren, der dazu verhilft, diese These plausibel zu machen. Ich möchte auch ergründen, was es für den Anspruch der juristischen Expertise bedeutet, wenn die unterstellte Einheit von praktischem Wissen und theoretischer Begründung sich dialektisch in die Differenz verkehrt. Die theoretische Begründung versichert dann nicht. Sie verunsichert. Sie bestätigt dem praktischen Wissen nicht, dass es ein Fundament hat, sondern belästigt es mit Fragen, über die sich kein Konsens herstellen lässt. ${ }^{8}$

Ich meine, dass wir uns in der Ära des nachpositivistischen Rechtsdenkens in einer solchen Situation befinden. ${ }^{9}$ Unter juristischen Experten verdichtet sich die Vermutung zur Gewissheit, dass so gut wie alles argumentierbar ist. ${ }^{10}$ Entscheidende Instanzen haben darin schon längst einen Freibrief erkannt, Begründungen durch Rechtsweistümer zu ersetzen, denen die Prämissen abgehen. ${ }^{11}$

Diese dialektische Verkehrung ist gefährlich. Sie lässt rechtliches Wissen arbiträr, benutzbar oder manipulierbar erscheinen. Das darf nicht sein. Zumindest darf das Wissen um diesen Wissenszustand für die Produktion von juristischer Expertise nicht relevant sein. Die Verkehrung muss kontrolliert werden. Die Begründung rechtlichen Wissens vermag das aber nicht zu leisten. Sie ist Teil des Problems. Sie erschließt zu viele denkbare Begründungsweisen. Leisten kann dies nur die juristische Expertise als Medium. Dafür muss sie aber ihre Gestalt verändern.

HABERMAS, Theorie des kommunikativen Handelns, Bd. 2: Zur Kritik der funktionalistischen Vernunft. Frankfurt am Main 1982, 536, und gilt insbesondere für die Unterscheidung des "Rechtsmediums" von der Festlegung von Kompetenzen, kollektiv verbindliche Entscheidungen zu treffen (Macht). Siehe JÜRGEN HABERMAS, Faktizität und Geltung. Beiträge zur Diskurstheorie des Rechts und des demokratischen Rechtsstaats. Frankfurt am Main 1992, 151-152, 159, 187.

${ }^{8}$ Siehe NIKLAS LUHMANN, Sthenographie und Euryalistik, in: HANS UlRICH GUMBRECHT/KARL LUdWIG PFEIFER (Hrsg.), Paradoxien, Dissonanzen, Zusammenbrïche. Situationen offener Epistemologie. Frankfurt am Main 1991, 58-82, hier: 75: „Jedes Begründen setzt sich durch seinen bloßen Vollzug (und auch durch den Vollzug einer Frage oder einer unendlichen Suche) dem Vergleich mit anderen Möglichkeiten und damit dem Selbstzweifel aus. Die Begründung produziert auf der Suche nach Notwendigkeiten Kontingenzen. Sie operiert kontraintuitiv. Sie entfernt sich von dem Ziel, das sie anstrebt. Sie sabotiert sich laufend selbst, indem sie einen Zugang zu anderen Möglichkeiten eröffnet, wo sie ihn verschließen möchte."

9 Siehe AlEXANDER SOMEK/NIKOLAUS FORGO, Nachpositivistisches Rechtsdenken. Inhalt und Form des positiven Rechts. Wien 1996.

${ }^{10}$ Siehe DUNCAN KENNEDY, A Critique of Adjudication. Cambridge (Mass.) 1999.

${ }^{11}$ Vor allem der EuGH hat, was die Abgabe von Rechtsweistümern angeht, einen beachtlichen "record". Siehe für ein Weistum aus jüngerer Zeit, Rs. C-184/99, Rudy Grzelczyk v Centre public d'aide sociale d'Ottignies-Louvain-la-Neuve Slg. 2001, I-06193. 
Dieser Gestaltveränderung sind die abschließenden Ausführungen zum Legalitätsglauben (Abschnitt 8) und zur elliptischen Generalisierung (Abschnitt 9) gewidmet.

\section{Die Grundidee}

In ihrer ursprünglichen Ausprägung versucht die Theorie der Steuerungsmedien aufzuklären, durch welche gesellschaftlich institutionalisierten Mechanismen Personen sich in die Lage versetzt sehen, das Verhalten anderer intentional zu steuern. ${ }^{12} \mathrm{Im}$ Ausgang von einer durch die eigene Theorieanlage bedingten Problemstellung untersucht Parsons, wie die Austauschbeziehungen zwischen den Subsystemen des sozialen Systems $\mathrm{zu}$ erfassen sind, ${ }^{13}$ in denen sich unterschiedliche Komponenten der Orientierung sozialen Handelns gegeneinander ausdifferenziert haben. ${ }^{14} \mathrm{Da}$ sich durch die Differenzierung der funktionsspezifischen Subsysteme (Kultur, Gemeinschaft, Politik und Wirtschaft) die Freiheitsgrade ${ }^{15}$ des Handelns erhöhen, ${ }^{16}$ ist im Rahmen der Theorie der Steuerungsmedien (in welcher die Medien wiederum ausdifferenzierten Subsystemen zugeordnet werden), ${ }^{17}$ auch aufzuzeigen, weshalb

12 Siehe Parsons, On the Concept of Political Power (Fn. 5), 312; Ders., On the Concept of Influence (Fn. 7), 361: "Such mechanisms are ways of structuring intentional attempts to bring about results by eliciting the response of others as actors to approaches, suggestions, etc."

13 Siehe dazu PARSONS, On the Concept of Political Power (Fn. 5), 306, 348-350; Ders., On the Concept of Influence (Fn. 7), 380 und bei RICHARD MÜNCH, Theorie des Handelns. Zur Rekonstruktion der Beiträge von Talcott Parsons, Emile Durkheim und Max Weber. Frankfurt am Main 1981, 123-124; HABERMAS, Theorie des kommunikativen Handelns, Bd. 2 (Fn. 7), 384; NIKL.AS LUHMANN, Einführende Bemerkungen zu einer Theorie der symbolisch generalisierten Steuerungsmedien, in: DERS., Soziologische Aufklärung, Bd. 2: Aufsätze zur Theorie der Gesellschaft. 2. Aufl., Opladen 1982, 170-192, hier: 171-172, 174; DERS., Die Wirtschaft der Gesellschaft. Frankfurt am Main 1988, 234.

14 Siehe dazu die Analyse der "Tiefenstruktur" der Parsonsschen Theorie bei MÜNCH, Theorie des Handelns (Fn. 13), 63, 67, 81-96.

${ }^{15}$ In ihrer Tiefenstruktur geben sie sich als doppelte Kontingenz zu erkennen. Siehe dazu LUHMANN, Einführende Bemerkungen zu einer Theorie der symbolisch generalisierten Steuerungsmedien (Fn. 13), 172; DERS., Die Wirtschaft der Gesellschaft (Fn. 13), 238.

${ }^{16}$ Siehe MüNCH, Theorie des Handelns (Fn. 13), 72, 76. Kritisch HabERMAS, Theorie des kommunikativen Handelns, Bd. 2 (Fn. 7), 333-338, 385.

${ }^{17}$ Siehe PARSONS, On the Concept of Political Power (Fn. 5), 353; $\mathrm{MUNCH}$, Theorie des Handelns (Fn. 13), 129, 135; LUHMANN, Einführende Bemerkungen zu einer Theorie der symbolisch generalisierten Steuerungsmedien (Fn. 13), 174. Siehe dazu, dass die Medien zum Aufbau von Subsystemen verwendet werden, HABERMAS, Theorie des kommunikativen Handelns, Bd. 2 (Fn. 7), 387-388; NIKLAS LUHMANN, Systemtheoretische Argumentationen. Eine Entgegnung auf Jürgen Habermas, in: JÜRGEN HABERMAS/ NIKLAS LUHMANN, Theorie der Gesellschaft oder Sozialtechnologie - Was leistet die Sys- 
bei zunehmender Wahlfreiheit der Akteure gleichwohl die Integration der Gesellschaft, die das gemeinsame Werk aller Subsysteme ist, möglich sein kann. ${ }^{18}$

Doch der ursprünglich struktur-funktionalistische ${ }^{19}$ Zuschnitt der Medientheorie soll uns im Folgenden nicht beschäftigen; von Interesse ist vor allem der generelle Situationstypus, in dem die Handlungskoordination auf Steuerungsmedien zurückgreift.

Erreicht die menschliche Kooperation ein Niveau, auf dem die interne Fusion von Situationsdeutungen und habitualisierten Verhaltensweisen zunehmend zerbricht, ${ }^{20}$ ist es nicht mehr möglich, die Handlungskoordination in die fraglose Reziprozität kollektiv geteilten Hintergrundwissens ${ }^{21}$ einzubetten. ${ }^{22}$ Verständigungsorientiertes Handeln ${ }^{23}$

temforschung? Frankfurt am Main 1971, 291-405, hier: 346; DERS., Einführende Bemerkungen zu einer Theorie der symbolisch generalisierten Steuerungsmedien (Fn. 13), 180; DERS., Die Wirtschaft der Gesellschaft (Fn. 13), 243.

${ }^{18}$ Siehe zur hierfür erforderlichen Grenzüberschreitung der Medien im Verhältnis zur Systemumwelt bei Parsons näher HABERMAS, Theorie des kommunikativen Handelns, Bd. 2 (Fn. 7), 399-400; MÜNCH, Theorie des Handelns (Fn. 13), 142.

${ }^{19}$ Der strukturell-funktionale Ansatz der Systemtheorie geht davon aus, dass Systeme über bestimmte Strukturen verfügen, um im Anschluss daran zu fragen, welche funktionalen Leistungen für den Erhalt dieser Strukturen erbracht werden müssen. Siehe dazu NIKLAS LUHMANN, Soziologie als Theorie sozialer Systeme, in: DERS., Soziologische Aufklärung, Bd. 1: Aufsätze zur Theorie sozialer Systeme. 4. Aufl., Opladen 1974, 113-136, hier: 113-114; HELMUT WILLKE, Systemtheorie. Eine Einführung in die Grundprobleme. Stuttgart 1982, 3 .

20 Siehe PARSONS, On the Concept of Influence (Fn. 7), 359; HABERMAS, Theorie des kommunikativen Handelns, Bd. 2 (Fn. 7), 273, 488. Siehe LUHMANN, Einführende Bemerkungen $z \mathfrak{u}$ einer Theorie der symbolisch generalisierten Steuerungsmedien (Fn. 13), 173, der insbesondere auf die Ausbreitung schriftlicher Kommunikation verweist. Siehe DERS., Die Wissenschaft der Gesellschaft. Frankfurt am Main 1990, 178-179: „Damit entfallen (...) diejenigen Verführungen, die zu einer sofortigen Annahme des Verstandenen verleiten. (...) Es muss zunächst unwahrscheinlich gewesen sein, dass man sich überhaupt zur Annahme einer Kommunikation als Prämisse weiteren Verhaltens entschließt, wenn die Situation nicht dazu verführt."

${ }^{21}$ Dieses Hintergrundwissen wird als ,organisierter Vorrat von Hintergrundannahmen" in der Lebenswelt lokalisiert. Siehe JÜRGEN HABERMAS, Erläuterungen zum Begriff des kommunikativen Handelns, in: DERS., Vorstudien und Ergänzungen zur Theorie des kommunikativen Handelns. Frankfurt am Main 1984, 571-606, hier: 591; DERS., Theorie des kommunikativen Handelns, Bd. 2 (Fn. 7), 189.

22 Siehe HABERMAS, Theorie des kommunikativen Handelns, Bd. 2 (Fn. 7), 393; GIESEN, Die Entdinglichung des Sozialen (Fn. 5), 224. Zur Kulmination dieser Entwicklung in der "Entdinglichung des Sozialen" siehe ebd., 142, 161-163.

${ }^{23}$ Siehe JÜRGEN HABERMAS, Theorie des kommunikativen Handelns, Bd. 1: Handlungsrationalität und gesellschaftliche Rationalisierung. Frankfurt am Main 1981, 128, 150. Der Begriff des kommunikativen Handelns bezieht sich auf die Tätigkeit von Aktoren, die versuchen, sich über die Handlungssituation zu verständigen, um darauf aufbauend ihre Handlungspläne einvernehmlich zu koordinieren. In der gemeinsamen Situationsdeutung wird die aktuelle Handlungssituation in einen vorgegebenen, von Hintergrundgewissheiten geprägten normativen Kontext eingebettet. $\mathrm{Zu}$ den mit dem Begriff des 
sieht sich mit dem Problem konfrontiert, dass die Konsensfindung durch die Erwartung resistenter Negationspotentiale belastet wird. ${ }^{24}$ Als alternativer Modus der Handlungskoordination überbrücken ${ }^{25}$ Steuerungsmedien die Interpretations- und Begründungsprozessen inhärenten Risiken, indem die Handlungskoordination von der Verständigung über Werte, Ziele und Handlungsmittel abgekoppelt und "gegenüber der Alternative von Einverständnis und fehlgeschlagener Verständigung neutralisiert" wird. ${ }^{26}$ Sie ermöglichen Koordination gerade dann, wenn von kontextgebundenem Hintergrundwissen abgesehen wird ${ }^{27}$ und Alter und Ego voneinander erwarten, dass sie aus beliebigen Gründen unterschiedliche Handlungspläne verfolgen mögen. Da die zu erwartende Inkongruenz ihrer Perspektiven ${ }^{28}$ in die gemeinsame Deutung dieser Situation aufgenommen wird, ${ }^{29}$ vermag die Inter-

kommunikativen Handelns einhergehenden einschlägigen Unterscheidungen siehe ebd. 385: „Eine erfolgsorientierte Handlung nennen wir instrumentell, wenn wir sie unter dem Aspekt der Befolgung technischer Handlungsregeln betrachten und den Wirkungsgrad einer Intervention in einem Zusammenhang von Zuständen und Ereignissen bewerten; strategisch nennen wir eine erfolgsorientierte Handlung, wenn wir sie unter dem Aspekt der Befolgung von Regeln rationaler Wahl betrachten und den Wirkungsgrad der Einflussnahme auf die Entscheidungen eines rationalen Gegenspielers bewerten. Instrumentelle Handlungen können mit sozialen Interaktionen verknüpft sein, strategische Handlungen stellen selbst soziale Handlungen dar. Hingegen spreche ich von kommunikativen Handlungen, wenn die Handlungspläne der beteiligten Aktoren nicht über egozentrische Erfolgskalküle, sondern über Akte der Verständigung koordiniert werden. Im kommunikativen Handeln sind die Beteiligten nicht primär am eigenen Erfolg orientiert; sie verfolgen ihre individuellen Ziele unter der Bedingung, dass sie ihre Handlungspläne auf der Grundlage gemeinsamer Situationsdefinitionen aufeinander abstimmen können. Insofern ist das Aushandeln von Situationsdefinitionen ein wesentlicher Bestandteil der für kommunikatives Handeln erforderlichen Interpretationsleistungen."

24 Siehe HABERMAS, Theorie des kommunikativen Handelns, Bd. 2 (Fn. 7), 393.

25 Habermas spricht in diesem Zusammenhang von der Substitution sprachlicher Handlungskoordination. Zwar werde durch Medien die für die Sprache charakteristische Verkörperung semantischer Gehalte einerseits (und zwar durch den Bezug zwischen generalisiertem Wert und realen Werten) und die Struktur von Geltungsanspruch und Einlösung (und zwar durch die Deckung des Handlungsvorschlags) andererseits nachgeahmt, allerdings gehe die rationale Binnenstruktur von Verständigung - also die Anerkennung kritisierbarer, in lebensweltlichen Kontexten eingebetteter Geltungsansprüche - verloren. Siehe HABERMAS, Theorie des kommunikativen Handelns, Bd. 2 (Fn. 7), 394.

${ }^{26}$ HABERMAS, Theorie des kommunikativen Handelns, Bd. 2 (Fn. 7), 393.

27 Siehe HABERMAS, Theorie des kommunikativen Handelns, Bd. 2 (Fn. 7), 394.

28 Das bedeutet natürlich "doppelte Kontingenz". Siehe LUHMANN, Einführende Bemerkungen $z u$ einer Theorie der symbolisch generalisierten Steuerungsmedien (Fn. 13), 171. "Soziale Systeme entstehen nur unter der Bedingung doppelter Kontingenz." (LUHMANN, Die Wirtschaft der Gesellschaft (Fn. 13), 237).

29 Siehe LUHMANN, Einführende Bemerkungen zu einer Theorie der symbolisch generalisierten Steuerungsmedien (Fn. 13), 172, 174; DERS., Die Wirtschaft der Gesellschaft (Fn. 
aktion auf einer selektiven Verknüpfung zu beruhen..$^{30}$ Die dabei vollzogene Repräsentation menschlicher Handlungsmotivation macht unterschiedliche Handlungspläne unter einem spezifischen Gesichtspunkt beobachtbar. ${ }^{31}$ Diese "mystifizierende", die Zusammengehörigkeit des Getrennten signalisierende Funktion übernehmen die Steuerungsmedien. ${ }^{32}$ Sie gestatten es, die Selektion Alters in eine Prämisse des Erlebens oder Handelns von Ego zu transformieren, ${ }^{33}$ indem sie von allen anderen Aspekten der Handlungsrationalität außer von der individuellen Erfolgsorientierung (in sozial generalisierter und sachlich spezifizierter Form) abstrahieren. Unter dem Vorzeichen von Erfolgsorientierung stellt sich das Koordinationsproblem demgemäß so dar, dass Alter Ego dazu zu bringen hat, eine Handlungssequenz in der von Alter vorgeschlagenen Art fortzusetzen, obwohl beide voneinander wissen, dass sie frei sind, $\mathrm{zu}$ handeln wie sie es wünschen..$^{34}$

Parsons entwickelt vor diesem Hintergrund ein Konditionierungsmodell, ${ }^{35}$ dessen "channel variable" darauf abstellt, ob Alter auf Egos Situation oder Intention einwirkt, während für die Kontrollvariable ein positiver und ein negativer Sanktionsmodus unterschieden wird. ${ }^{36}$ Daraus leitet Parsons ein Grundschema der Medienkommunikation ab. ${ }^{37}$ Als

13), 237-238. Systemtheoretisch betrachtet bedeutet dies, dass die Kontingenz individuellen Handelns im System selbst „abgebildet” wird. Siehe LUHMANN, Einführende Bemerkungen $z u$ einer Theorie der symbolisch generalisierten Steuerungsmedien (Fn. 13), 172. Siehe freilich $\mathrm{zu}$ den Unterschieden zur auch hier angestrengten handlungstheoretischen Medienanalyse bei HABERMAS, Theorie des kommunikativen Handelns, Bd. 2 (Fn. 7), 390-391.

30 Siehe LUHMANN, Einführende Bemerkungen zu einer Theorie der symbolisch generalisierten Steuerungsmedien (Fn. 13), 174.

31 Es handelt sich dabei natürlich um eine Beobachtung "zweiter Ordnung", also um eine Beobachtung von Beobachtungen. Siehe LUHMANN, Die Wissenschaft der Gesellschaft (Fn. 20), 87.

32 Siehe LUHMANN, Die Wissenschaft der Gesellschaft (Fn. 20), 189-190.

33 Siehe LuHMANN, Systemtheoretische Argumentationen. Eine Entgegnung auf Jürgen Habermas (Fn. 17), 344.

34 Siehe HABERMAS, Theorie des kommunikativen Handelns, Bd. 2 (Fn. 7), 413-414.

35 Siehe dazu im Kontext mit den "pattern variables" MÜNCH, Theorie des Handelns (Fn. 13), 77-87.

36 Siehe PARSONS, On the Concept of Political Power (Fn. 5), 309-311; DERs., On the Concept of Influence (Fn. 7), 361-363; LUHMANN, Einführende Bemerkungen zu einer Theorie der symbolisch generalisierten Steuerungsmedien (Fn. 13), 174; MüNCH, Theorie des Handelns (Fn. 13), 127, 129, 135; HABERMAS, Theorie des kommunikativen Handelns, Bd. 2 (Fn. 7), 414.

37 Siehe zur (problematischen) Weiterentwicklung der Medientheorie durch Übertragung des Grundschemas auf andere „Träger" etwa bei TALCOTT PARSONS, Social Structure and the Symbolic Media of Interchange, in: PETER M. BLAU (Hrsg.), Approaches to the Study of Social Structure. New York 1975, 94-120, 104-120; HABERMAS, Theorie des kommunikativen Handelns, Bd. 2 (Fn. 7), 386-387 und zum Beispiel die Analyse des 
Mittel des Anreizes (inducement) ist Geld im Wirtschaftssystem verankert und wirkt positiv auf die Situation des Handelnden ein, um ihn zur Ausführung einer bestimmten Handlung zu motivieren. Macht ist (als Mittel der Abschreckung) ${ }^{38}$ im politischen System institutionalisiert und wirkt negativ auf die Handlungssituation, um von der Ausführung einer bestimmten Handlung abzuhalten. Der im Gemeinschaftssystem beheimatete Einfluss wirkt als Mittel der Überredung und Begründung von "Gemeinschaft" ${ }^{\prime 3}$ positiv auf die Einstellung des Handelnden, wohingegen die als Mittel der Aktivierung von moralischen Haltungen im sozial-kulturellen System verwurzelte Wertbindung (moralische Autorität) mit einem negativen Sanktionsmodus (d. h. durch die Evokation von Schuldgefühlen) das Verhalten des Adressaten steuert. $^{40}$

In der Situationsdeutung, die dem Konditionierungsschema zugrunde liegt, verfolgen Alter und Ego keine gemeinsam akkordierten oder

"Lebensstils" und "methodischer Argumente" als Medien der Kommunikation bei GIESEN, Die Entdinglichung des Sozialen (Fn. 5), 234-239. Zu einer übermäßigen Ausrichtung der Analyse am Musterbeispiel des Geldes siehe insbesondere RICHARD MüNCH, Dialektik der Kommunikationsgesellschaft. Frankfurt am Main 1991, 103-108, 116-132, 342-345.

${ }^{38} \mathrm{Zu}$ ergänzen ist freilich, dass dieses Mittel nur im Rahmen von bestimmten normativen und institutionellen Prämissen eingesetzt werden darf. Um Missverständnissen vorzubeugen, verweist Parsons daher darauf, dass Macht nicht schlicht ein Mittel der Abschreckung sei, „(...) but rather of mobilizing the performance of binding obligations, with the conditional implication of the imposition of negative sanctions (...)." So PARSONS, On the Concept of Influence (Fn. 7), 363.

${ }^{39}$ Siehe PARSONS, On the Concept of Influence (Fn. 7), 370: ,... [A]ttempting to influence is to a degree an attempt to establish a common bond of solidarity ... to constitute a 'we'."

40 Siehe, was die beiden letzteren Medien des Konditionierungsschemas betrifft, die zutreffende Kritik bei HABERMAS, Theorie des kommunikativen Handelns, Bd. 2 (Fn. 7), 415-416. Im Rahmen von Parsons Systemtheorie bedeutet dies, dass der Handelnde dazu bewogen werden soll, vermittels der Medien die mit der spezifischen Funktion eines Subsystems verbundene Orientierung zu übernehmen, indem er wie vorgeschlagen handelt (MUNNCH, Theorie des Handelns (Fn. 13), 134). Thre Verankerung in einzelnen Subsystemen bewahrt somit Spezifikation bei gleichzeitig stattfindender wechselseitiger Durchdringung und Begrenzung im Wege der Zirkulation. Münch erläutert dies am Beispiel des Geldes folgendermaßen: „[Die] Zirkulierbarkeit [der Medien] erlaubt das Eindringen ökonomischer Anforderungen in das kulturelle System und die Übertragung kultureller Anforderungen auf das ökonomische System." (ebd., 135). Da die Konsumentennachfrage auf kulturelle Standards zurückgeht, produzieren Unternehmen unter kulturell eingeschränkten Bedingungen, die sie sich gleichwohl im Medium des Geldes (der Nachfrage) verbindlich machen. In der medienvermittelten "Interpenetration" zwischen Subsystemen finden daher einerseits wechselseitige Austauschbeziehungen (etwa zwischen Geld und Macht im Verhältnis von ökonomischem und politischem System) und andererseits Überformungen der Materie des Handelns im Lichte der im Austausch stehenden Systeme statt, ohne dass der erreichte Grad der Systemdifferenzierung zurückgenommen werden müsste (näher ebd., 135-143). 
durch kontextspezifische Interpretationen gegeneinander abgestimmten Handlungspläne. Die Interaktion wird allein deshalb möglich, weil die Art, in der Alter einen Handlungsvorschlag macht, unabhängig von Alters Beweggründen für Ego ein Motiv darstellen kann, diesen Handlungsvorschlag anzunehmen.41 Diese Integration von Alters Selektion und Egos Motivation überbrückt doppelte Kontingenz. ${ }^{42}$ Die Handlungskoordination wird vom Deutungs- und Begründungsaufwand entlastet. Vermöge der Indifferenz individueller Lebenslagen oder Bedürfnisartikulationen kann Alter sein Wollen durch das symbolische Medium durchscheinen lassen, ohne Gründe darlegen zu müssen. Denn nicht der (etwa aus seiner Lebenslage verständliche) Grund für die Interaktionsaufforderung an Ego, sondern allein die Modalität, seinen Willen zu artikulieren, ist dazu angetan, Ego zur Ausführung einer Anschlusshandlung zu motivieren:

"Von Steuerungsmedien wollen wir immer dann sprechen, wenn durch die Art der Selektion zur Annahme motiviert wird, wenn also die Selektionsweise zugleich als Motivationsstruktur fungiert."43

\section{Komponenten legitimer Konditionierung}

Die faktischen und normativen Voraussetzungen, auf denen das Konditionierungsschema aufbaut, 44 erschließen sich nicht zufällig mit Blick auf die Bedingungen, unter denen Symbole semantische und pragmatische Effekte entfalten. Obwohl ihre konventionell feststehende illokutionäre Rolle von kommunikativen Inhalten und lokalen Kontexten weitestgehend unabhängig (Generalisierung) ist, ${ }^{45}$ sind sie nach dem Ty-

${ }^{41}$ Siehe LuHManN, Einführende Bemerkungen zu einer Theorie der symbolisch generalisierten Steuerungsmedien (Fn. 13), 175-176, 185.

42 Siehe LuHMANN, Die Wirtschaft der Gesellschaft (Fn. 13), 238

${ }^{43}$ LUHMANN, Systemtheoretische Argumentationen (Fn. 17), 345. Die Interaktion vermag zu gelingen, wenn im Symbol zum Ausdruck gebracht wird, dass die Selektionsart zur Prämisse von Egos eigener Selektion werden kann.

44 Unmittelbar einleuchtend ist das Schema daher auch wohl nur unter den Standardbedingungen des Geldes (wie instrumentelle Handlungsorientierung, metrische Standardisierung, Zirkulationsfähigkeit als Ressource, Knappheit, physische Kontrolle über Deckungsreserven, normative Verankerung und "marktförmige" Interaktionsstrukturen). Sie sind schon für das Machtmedium nicht in vollem Umfang gegeben. Siehe dazu GIESEN, Die Entdinglichung des Sozialen (Fn. 5), 230, 240-241; HABERMAS, Theorie des kommunikativen Handelns, Bd. 2 (Fn. 7), 402-403, 406-407. Bekanntlich hat Parsons sein Konzept im Ausgang von einer Analyse des Wirtschaftssystems entwickelt. Siehe PARSONS, On the Concept of Political Power (Fn. 5), 300-302, 323.

${ }^{45}$ Siehe PARSONS, On the Concept of Political Power (Fn. 5), 308; MŨNCH, Theorie des Handelns (Fn. 13), 125. 
pus von Interaktion spezifiziert, für den sie Relevanz besitzen. ${ }^{46}$ Pragmatisch betrachtet sind Medien signifikante Symbole, ${ }^{47}$ da Ego durch ein bestimmtes Zeichen (die "Art der Selektion" Alters, d. h. ein Angebot, eine Weisung, einen Rat, eine Ermahnung) gleichsam automatisch (durch einen dynamischen Interpretanten) ${ }^{48}$ auf das Motiv gestoßen wird, wie vorgeschlagen zu handeln. Auf das Motiv stößt Ego vermöge der sozialen Situationsdeutung, auf der mediale Kommunikation beruht. Sie sagt etwas aus über den Handlungserfolg, an dem Alter orientiert ist. Über die Bedeutung dieses Erfolgs gibt - je nach dem Grad der Ausdifferenzierung des Mediums - entweder ein sozial generalisierter Wert der Verhaltensorientierung oder eine Konsequenz ("Deckung") den Ausschlag, die für die Annahme des Verhaltensangebots in Aussicht gestellt wird.

Dem Medium kommt damit in einer Relation seine symbolische Funktion zu, in welcher Alter versucht, das Verhalten Egos strategisch $z$ steuern. ${ }^{49}$ Es ist unerheblich, ob Alter selbst an dem unterstellten generalisierten Wert (oder der Deckung) orientiert ist. ${ }^{50} \mathrm{Da}$ in der sozialen

${ }^{46}$ Siehe PARSONS, Social Structure and the Symbolic Media of Interchange (Fn. 37), 96; LUHMANN, Die Wirtschaft der Gesellschaft (Fn. 13), 239.

${ }^{47}$ Nach Mead werden in sozialen Akten die Reaktionen der Empfänger eines signifikanten Reizes bei der Emission dieses Reizes antizipiert. Die Situation gemeinsamen Handelns lässt sich damit vor und bei dessen Ausführung präsent halten. Siehe GEORGE HERBERT MEAD, Eine behavioristische Erklärung des signifikanten Symbols (1922), dt. in: DERS., Gesammelte Aufsätze. Herausgegeben von HANS JOAS. Bd. 1. Frankfurt am Main 1980, 290-298, hier: 296-297.

${ }^{48}$ Ein dynamischer Interpretant besteht aus der Wirkung, die ein Zeichenmittel beim Empfänger auslöst. Siehe CHARLES S. PEIRCE, Ein Überblick über den Pragmatizismus (1907), dt. in: DERS., Schriften zum Pragmatismus und Pragmatizismus. Herausgegeben von KARLOTTO APEL, 2. Aufl., Frankfurt am Main 1976, 498-532, hier: 512-513 (§ 5.475).

49 Darauf weist PARSONS, On the Concept of Political Power (Fn. 5), 306, hin, wenn er sagt, dass Geld nur "im Austausch" Nützlichkeit bezeichnet. Siehe auch HaBERMAS, Theorie des kommunikativen Handelns, Bd. 2 (Fn. 7), 413. Die Konditionierung nach dem Reiz-Reaktions-Schema wäre unmöglich, würde nicht die Orientierung am generalisierten Wert die Überbrückung divergenter Handlungsperspektiven im Steuerungsvorgang gewährleisten.

50 Daraus erklärt sich das alltägliche, im Rahmen der bisherigen Theorieansätze missverständlich als "Zirkulation" bezeichnete Phänomen, dass sich Geld als Steuerungsmedium auch zum Zweck der effektiven Erreichung eines gemeinsamen Ziels oder Macht zur Beschaffung von Geld einsetzen lässt (siehe PARSONS, On the Concept of Political Power (Fn. 5), 306). Die "Konversion" von Macht in Geld (etwa durch Besteuerung) betrifft die Beschaffung von Steuerungsressourcen, die auf verschiedensten Wegen vor sich gehen kann (auch ohne Medien, etwa durch Raub), nicht aber den gleichsam dinghaften vorliegenden Bedeutungsgehalt eines Mediums (siehe aber PARSONS, On the Concept of Influence (Fn. 7), 380). Was ein Medium (als Symbol) bedeutet, ergibt sich aus der Perspektive des Adressaten. Wir werden unter dem Titel der Koppelung von Medien auf dieses Problem zurückkommen. 
Situationsdeutung, welche den Mediengebrauch ermöglicht, ${ }^{51}$ bloß auf die Orientierung des Adressaten abgestellt wird, beruht der Gebrauch von Steuerungsmedien auf einer grundlegenden Asymmetrie (die sich freilich in Situationen wechselseitiger Steuerung mit demselben Medium wiederum ausgleicht). Die Situation wird derart repräsentiert, dass autonome, auf ihren eigenen Handlungserfolg bedachte Akteure einander gegenüberstehen, ${ }^{52}$ von denen einer versucht, die Entscheidung des anderen zu steuern..$^{53}$

In der Situationsdeutung wird die Bedeutung von "Handlungserfolg" unter Abstraktion von individuellen Handlungsplänen bestimmt. Wenigstens für Ego ist zu unterstellen, dass er oder sie sich an einem sozial generalisierten Wert orientiert, ${ }^{54}$ der bei der Annahme des Handlungsvorschlages realisiert wird. Dieser durch das Medium in Aussicht gestellte ${ }^{55}$ Konkretisierungsvorgang kann, wie wir noch sehen werden, durch Deckungen substituiert oder überlagert werden. ${ }^{56}$ Alter muss also zur Verhaltenssteuerung an den sozial generalisierten Wert (oder Unwert) eines individuellen Handlungserfolgs appellieren. Beim Geld ist das, laut Parsons, der Nutzen, bei der Macht die Effektivität der gemeinschaftlichen Zielerreichung, beim Einfluss die Loyalität mit einer Gruppe, und bei der Wertbindung die Integrität der moralischen Ordnung. ${ }^{57}$ So ist für den Gebrauch des Geldmediums vorauszusetzen, dass Ego das Verhältnis von individuellem Handlungsziel und rentablen

51 Siehe zum folgenden PARSONS, On the Concept of Influence (Fn. 7), 358-360; HABERMAS, Theorie des kommunikativen Handelns, Bd. 2 (Fn. 7), 406-407; GieseN, Die Entdinglichung des Sozialen (Fn. 5), 230, 240-241.

52 Siehe HABERMAS, Theorie des kommunikativen Handelns, Bd. 2 (Fn. 7), 401.

53 Siehe, wenn auch unter Vorzeichen der Symmetrie formuliert, HABERMAS, Theorie des kommunikativen Handelns, Bd. 2 (Fn. 7), 413-414, 418; GIESEN, Die Entdinglichung des Sozialen (Fn. 5), 230. Geld fungiert als Medium in bestimmten Situationen (die Standardsituation ist der Gütertausch), in der Alter unterstellt, dass Ego seine Entscheidungen ausschließlich vom eigenen Handlungsziel abhängig macht. Siehe HABERMAS, Theorie des kommunikativen Handelns, Bd. 2 (Fn. 7), 395. Konsensbildung über ein gemeinsames Ziel kommt hier nicht in Betracht.

54 Siehe MÜNCH, Theorie des Handelns (Fn. 13), 127-128.

55 Genau besehen, wird dieser Zusammenhang der Konkretisierung durch das Medium (etwa Geldsummen im Rahmen eines Angebots) bezeichnet. Es war daher von Anfang an völlig verkehrt anzunehmen, Medien stünden für "the real thing", es handle sich dabei um ein Gut, eine Dienstleistung oder eine andere Handlung. So aber PARSONS, On the Concept of Influence (Fn. 7), 358; MUNCH, Recht als Medium der Kommunikation (Fn. 7), 79-80; DERS., Theorie des Handelns (Fn. 13), 125; HABERMAS, Theorie des kommunikativen Handelns, Bd. 2 (Fn. 7), 406; GEPHART, Gesellschaftstheorie und Recht (Fn. 6), 247-248. Das Geld symbolisiert Nützlichkeit für den Adressaten. Wie sich ihm diese konkretisiert, ist gleichgültig.

56 Siehe dazu unten S. 420

57 Siehe PARSONS, On the Concept of Influence (Fn. 7), 376; HABERMAS, Theorie des kommunikativen Handelns, Bd. 2 (Fn. 6), 409. 
Handlungsmitteln zwar autonom, ${ }^{58}$ gleichwohl aber unter Orientierung am generalisierten Wert der Nützlichkeit bestimmt. ${ }^{59}$ Unter dieser Bedingung ist es möglich, Interaktionsangebote relativ exakt $\mathrm{zu}$ bewerten, ${ }^{60}$ weil Geld aus der Perspektive des Adressaten über eine "eingebaute Präferenzstruktur" ${ }^{\prime \prime 1}$ verfügt. Durch das signifikante Symbol wird der Sender in die Lage versetzt, die in Adressatenperspektive stattfindende Koppelung von Information und Motivation zur Verhaltenssteuerung heranzuziehen, ${ }^{62}$ ohne dass ein Einverständnis über die Richtigkeit oder Dienlichkeit der Transaktion erzielt werden muss.

Aus dem Vorgehenden ist unschwer zu erkennen, dass die Initialisierung solcher medialer Symbole auf einer Situationsdeutung beruht. Deren Bedeutung und Maßgeblichkeit ergibt sich aus dem institutionellen Code des Mediums. ${ }^{63}$ Dieser besteht aus normativen Regeln, die den Anwendungsbereich der Medien abgrenzen und die Bedingungen der Medienkommunikation konstituieren und regulieren. ${ }^{64}$ Parsons spricht in diesem Zusammenhang von "institutions":

„Institutions (...) are complexes of normative rules and principles which, either through law or other mechanisms of social control, serve to regulate social action and relationships - of course, with varying degrees of success." 65

Er bezeichnet Eigentum (und Vertrag) als den Code, der Besitzrechte festlegt, ${ }^{66}$ Autorität als den Code, der Ermächtigungen zur kollektiv ver-

58 Die Beteiligten haben voneinander das Einnehmen einer objektivierenden Einstellung zu erwarten. Siehe HABERMAS, Theorie des kommunikativen Handelns, Bd. 2 (Fn. 7) 396. Was es bedeutet, dass der Adressat "frei" sei, erfährt hierbei insofern eine Spezifikation, da Freiheit als Vermögen bestimmt wird, den individuellen Nutzen (im Sinne der Optimierung des Verhältnisses von Aufwand und Ertrag) kraft eigener Vorlieben und aufgrund eigener Kalkulationen zu bestimmen und in Geldsummen auszudrücken.

59 Siehe GIESEN, Die Entdinglichung des Sozialen (Fn. 5), 227; HABERMAS, Theorie des kommunikativen Handelns, Bd. 2 (Fn. 7), 395; MüNCH, Theorie des Handelns (Fn. 13), 127.

60 Siehe dazu vor allem GIESEN, Die Entdinglichung des Sozialen (Fn. 5), 225.

61 Siehe HABERMAS, Theorie des kommunikativen Handelns, Bd. 2 (Fn. 7), 395; GIESEN, Die Entdinglichung des Sozialen (Fn. 5), 225.

62 Siehe auch LUHMANN, Einführende Bemerkungen zu einer Theorie der symbolisch generalisierten Steuerungsmedien (Fn. 13), 175-176.

63 Siehe MÜNCH, Theorie des Handelns (Fn. 13), 126; HABERMAS, Theorie des kommunikativen Handelns, Bd. 2 (Fn. 7), 402.

64 Siehe PARSONS, On the Concept of Political Power (Fn. 5), 319-320; MUNCH. Theorie des Handelns (Fn. 13), 125-126; HABERMAS, Theorie des kommunikativen Handelns, Bd. 2 (Fn. 7), 398-399, 402

65 PARSONS, Social Structure and the Symbolic Media of Interchange (Fn. 37), 97.

66 Siehe etwa PARSONS, On the Concept of Political Power (Fn. 5), 320. 
bindlichen Normerzeugung enthält, ${ }^{67}$ die relative Ordnung des Prestiges als Code für die Mobilisierung der Zustimmung von Angehörigen einer Gruppe ${ }^{68}$ und die moralische Führung als den Code der Wertbindung. ${ }^{69}$ Vom institutionellen Code ist das Medium als "message" zu unterscheiden, in welcher Codebedeutungen in einzelnen Sprechakten aktualisiert werden ${ }^{70}$ (wobei ersterer den Ausschlag über den "Wert" der Botschaft gibt). ${ }^{71}$

Die latente Akzeptanz der einzelnen institutionellen Codes ist unabdingbar. ${ }^{72}$ Sonst würden Medien nicht funktionieren. Gerade damit die gegenüber ihren eigenen normativen Grundlagen gleichgültige Konditionierung sozial akzeptabel sein kann, hat ihre Legitimität festzuste-

67 "This may be defined as the legitimate capacity to make and to contribute to the implementations of decisions which are binding on a specifiable collective unit or class of them, where the holder of authority has some kind of right of speaking in the name of that collectivity. ... [A] uthority is the institutionalized code within which the language of power' is meaningful and, therefore, its use will be accepted in the requisite community ...." So PARSONS, Social Structure and the Symbolic Media of Interchange (Fn. 37), 98; DERS., On the Concept of Political Power (Fn. 5), 331.

68 Siehe PARSONS, Social Structure and the Symbolic Media of Interchange (Fn. 37), 104. Die Position in einer solchen Prestigeordnung verleiht nach Parsons das Recht, eine Aussage zu machen, ohne deren Gehalt verifizieren zu müssen. Siehe PARSONS, On the Concept of Influence (Fn. 7), 369. Das Bestehen einer "Prestigeordnung", in der Einfluss verankert sein könnte, scheint freilich mit denjenigen, die über Einfluss verfügen zusammenzufallen, und würde, gäbe es sie, in demokratischen Gesellschaften das Dissensrisiko nicht verringern, sondern vergrößern. Ihre Institutionalisierung muss daher, sofern sie nicht mit einer machtförmigen Ämterhierarchie verbunden ist, in der Tat fragwürdig erscheinen. Siehe dazu HABERMAS, Theorie des kommunikativen Handelns, Bd. 2 (Fn. 7), 410-411 (dort auch zum Ausnahmefall Wissenschaft).

69 Siehe PARSONS, On the Concept of Political Power (Fn. 5), 353. Auch hier mag es problematisch erscheinen, ob die moralische Autorität derjenigen, die durch Ermahnungen an Wertbindungen erinnern, sollte überhaupt Konsens darüber bestehen, nicht mit deren Träger zusammenfällt. Dagegen findet Macht in der Tat in verschiedensten öffentlich-rechtlichen und privatrechtlichen Normen eine institutionelle Grundlage. Siehe hiezu (wenn auch unter Auslassung des Privatrechts) HABERMAS, Theorie des kommunikativen Handelns, Bd. 2 (Fn. 7), 404.

70 Siehe zum dahinter stehenden linguistischen Modell PARSONS, On the Concept of Influence (Fn. 7), 357.

${ }^{71}$ Nach der in Geld gemessenen quantitativen Menge an Eigentum bemisst sich der Wert eines Angebotes. Anders wirkt der institutionelle Code für das Medium Macht: Der "Wert" der Kommunikation bemisst sich aus dem Verhältnis von Sender und Empfänger. Ansonsten ist die Quantifizierung beim Machtmedium ohnedies nur analog durchzufuihren. Siehe PARSONS, On the Concept of Political Power (Fn. 5), 317; HABERMAS, Theorie des kommunikativen Handelns, Bd. 2 (Fn. 7), 402.

72 Siehe dazu auch HABERMAS, Theorie des kommunikativen Handelns, Bd. 2 (Fn. 7), 399, $404,407$. 
hen. Überdies obwaltet in der Medienkommunikation eine ,organische List $^{\prime \prime},{ }^{73}$ die von Parsons folgendermaßen beschrieben worden ist:

"[F]rom the point of view of the acting unit, whether it be individual or collectivity, there is one 'direction' in which the medium serves as a means of furthering its interests, and this includes the structuring of conditions under which, in various contingencies, its interest is more or less secure. On the other hand, what from an acting unit's point of view are certain norms or rules to which it is subject in furthering its interest are, from the point of view of the system, a set of conditions under which process in it can be carried on stably, without disturbance to its integration and other functions. ${ }^{\prime 74}$

Für die Institutionalisierung von Medieninteraktionen lässt sich demnach also ein legitimes gemeinsames Interesse ${ }^{75}$ benennen (Gesamtwohlfahrt, ${ }^{76}$ Koordination gemeinschaftlichen Handelns zum Zwecke der Verwirklichung gemeinsamer Ziele, ${ }^{77}$ Freiheit der Assoziation ${ }^{78} \mathrm{Er}-$ haltung der moralischen Ordnung). In der Medienkommunikation waltet indes insofern eine organische List, als das gemeinsame Interesse durch individuelles erfolgsorientiertes realisiert wird. Um dies zu gewährleisten, müssen durch die jeweiligen institutionellen Codes orientierungsspezifische Deckungen (backings) festgelegt werden. ${ }^{79}$ Den für die mediengesteuerte Interaktion zentralen Zusammenhang von Legitimation durch ein gemeinsames Interesse, institutionellen Code und Deckung hat Parsons selbst in seiner Definition des Machtmediums deutlich zum Ausdruck gebracht:

"Power $(. .$.$) is the generalized capacity to secure the performance of binding$ obligations by units in a system of collective organization when the obligations are legitimized with reference to their bearing on collective goals and

\footnotetext{
73 Siehe Friedrich Wilhelm Joseph von SCHELling, Ideen zu einer Philosophie der Natur (1797), in: Sämmtiche Werke. Herausgegeben von KARL. FRIEDRICH AUGUST SCHELLING. Stuttgart 1856-1861, Bd. I/2, 40-41.

74 PARSONS, On the Concept of Influence (Fn. 7), 364-365.

${ }^{75}$ Im Gegensatz zu Parsons' auf die faktische Akzeptanz von Ordnungen abzielenden Legitimationsbegriff wird Legitimation hier normativ verstanden. Siehe aber PARSONS, On the Concept of Influence (Fn. 7), 364, 366.

76 "Institutionalized money has the advantage of a far wider usefulness in exchange than ... goods [that are subject to deterioration]." $\mathrm{Zu}$ dieser Form der Legitimation der auf dem Geldmedium aufbauenden Interaktion siehe bei PARSONS, On the Concept of Influence (Fn. 7), 365 und CRAWFord BROUGH MACPHERSON, Die politische Theorie des Besitzindiwidualismus. 2. Aufl., Frankfurt am Main 1980, 268-294.

${ }^{77}$ Siehe zur Rechtfertigung der Institutionalisierung von Macht zum Zwecke der Realisierung von Solidarität bei PARSONs, On the Concept of Political Power (Fn. 5), 312, $327-328$.

78 Siehe zu dieser Legitimation des Einflusses bei PARSONS, On the Concept of Influence (Fn. 7), 368.

79 Siehe PARSONS, On the Concept of Influence (Fn. 7), 365
} 
where in case of recalcitrance there is a presumption of enforcement by negative situational sanctions-what ever the agency of that enforcement." 80

Die rechtliche Institutionalisierung von Macht ist legitim, wenn und insofern sie der Verfolgung von gemeinsamen Zielen dient. Deckung erhält die befehlende Ausübung von Macht durch die Androhung von Sanktionen. Diese Deckung ist aber auch nur insoweit akzeptabel, als sie dazu hinreicht, den generalisierten Wert der gemeinsamen Zielerreichung zu realisieren.

\section{Legitimation und Resymmetrierung}

Aus der Perspektive des Adressaten ist es riskant, sich durch ein Steuerungsmedium lenken zu lassen. ${ }^{81}$ Aufgrund der situationsimmanenten Asymmetrie ist die Realisation des generalisierten Wertes nicht gesichert. $^{82}$ Im Steuerungsmedium wird sie zunächst aufgeschoben. ${ }^{83}$ Durch die Ausübung von Einfluss sollen Überzeugungen hervorgerufen werden, indem von einer Begründung Abstand genommen und pauschal auf vorhandenes Wissen verwiesen wird. Das Geld wird zur Verfügung über nützliche Dinge herangezogen, obwohl es selbst keinen realen Wert hat. Einem Befehl wird gehorcht, obwohl in ihm nicht durchsichtig gemacht wird, weshalb die Befolgung zur Verwirklichung eines kollektiven Zieles beiträgt. Die Realisation des generalisierten Wertes kann daher nur gewährleistet sein, wenn Resymmetrierungen durchgeführt werden. Geld hat nicht nur vom Adressaten, sondern reziprok auch vom Sender akzeptiert und in einem stabilen Währungssystem verankert zu werden. Der Befehlsgeber hat sich von der Akzeptanz des Befehlsempfängers abhängig zu machen. Resymmetrierungen dieser Art werden im institutionellen Code vollzogen. Sie lassen sich abermals mit Konditionierungsstrategien verbinden und durch Modalitäten der Deckung ${ }^{84}$ faktisch implementieren. Die asymmetrische Steue-

80 PARSONS, On the Concept of Political Power (Fn. 5), 308

81 Siehe dazu auch PARSONS, On the Concept of Influence (Fn. 7), 360; DERS., On the Concept of Political Power (Fn. 5), 314.

82 Siehe PARSONS, On the Concept of Political Power (Fn. 5), 308-309.

83 Anders formuliert: Sie unterbrechen den Zusammenhang von Anspruch und Einlösung. Zur Rekonstruktion der Medien aus dieser Perspektive siehe HABERMAS, Theorie des kommunikativen Handelns, Bd. 2 (Fn. 7), 397-398.

84 Siehe im Unterschied zur alltagssprachlichen Handlungskoordinierung mittels Gründen, die wegen ihrer internen Beziehung zum Geltungsanspruch eine motivierende Kraft entfalten, sowie dazu, dass die Alltagssprache - anders als Medien - keiner weiteren Beglaubigung bedarf, bei HABERMAS, Theorie des kommunikativen Handelns, Bd. 2 (Fn. 7), 398. 
rungssituation wird damit auf einer höheren Ebene ausgeglichen. Die Koppelung von Konditionierung und Deckung, auf der dieser Ausgleich beruht, kann freilich nur dann als allgemein akzeptabel gelten, wenn der institutionelle Code das Zusammenspiel von Deckung und generalisiertem Wert dauerhaft gewährleistet. Es muss, mit anderen Worten, durchsichtig sein, weshalb die Deckung von Befehlen mit Sanktionspotentialen der kollektiven Zielerreichung dient. Andernfalls würde das Steuerungsmedium nicht allgemein akzeptiert.

An der Reichweite dieses Zusammenspiels bemisst sich der Grad der Ausdifferenzierung von Medien. Sie ist umso vollständiger, je umfassender ein Medium bei der Gestaltung des legitimierenden institutionellen Codes auf andere Steuerungsmedien zurückgreift (siehe Tab. 2). Daraus ergibt sich ein Verhältnis interner Verweisungen, das, wie wir sehen werden, schließlich zu einem System der wechselseitigen Konstitution gerinnt.

Für das Geldmedium lässt sich die erforderliche Resymmetrierung durch die reziproke Institutionalisierung eines stabilen Währungssystems durchführen, ${ }^{85}$ das den Zugang zu Deckungsreserven (dem akzeptierten Tauschmittel) im Rahmen eines allgemeinen Systems des Güteraustauschs sicherstellt. ${ }^{86} \mathrm{In}$ ihm wird auch die Zusammenführung der Perspektiven von generalisierter Wertorientierung und Deckung vollzogen. Sie ist unproblematisch, da für beide die individuelle Nutzenorientierung maßgeblich ist. Ein stabiles Währungssystem macht den Gebrauch des Geldes allgemein akzeptabel. Die Unauffälligkeit dieses Zusammenspiels ist der Effizienz des Mediums zuträglich. ${ }^{87}$ Darüber hinaus kann Geld nicht nur gemessen, sondern auch in beliebigen Größen veräußert oder gespeichert und somit relativ flexibel als Mittel der strategischen Handlungskoordination eingesetzt werden. ${ }^{88}$ Der Geldwert stellt eine Größe dar, in der sich der Wert von Waren (bzw. Tauschgewinnen) ziffernmäßig ausdrücken lässt. ${ }^{89}$

\footnotetext{
85 Siehe HABERMAS, Theorie des kommunikativen Handelns, Bd. 2 (Fn. 7), 398, 406. Gerät das Geld angesichts inflationärer Entwicklungen in ein Missverhältnis zu den realen Werten, so hat das dementsprechende Vertrauensverluste für das Medium insgesamt zur Folge. Siehe GIESEN, Die Entdinglichung des Sozialen (Fn. 5), 227.

${ }^{86}$ Zur Paradoxie des Geldes, als bloßer Wertausdruck der Waren selbst wiederum im Währungssystem einen Wert zugespielt zu erhalten, siehe schon GEORG SIMMEL, Philosophie des Geldes (2. Aufl. 1907), in: Gesamtausgabe. Bd. 6. Herausgegeben von DAVID P. FRISBY/KLAUS CHRISTIAN KÖHINKE. Frankfurt am Main 1989, 126-127.

${ }^{87}$ Siehe zur Nutzenorientierung GIESEN, Die Entdinglichung des Sozialen (Fn. 5), 226, 230.

${ }^{88}$ Siehe HABERMAS, Theorie des kommunikativen Handelns, Bd. 2 (Fn. 7), 396.

${ }^{89}$ Siehe ebd., 397.
} 


\begin{tabular}{|c|c|c|c|c|}
\hline & Geld & Macht & Einfluss & Wertbindung \\
\hline Steuerungsvariable & $\begin{array}{l}\text { situations- } \\
\text { gebunden }\end{array}$ & $\begin{array}{l}\text { situations- } \\
\text { gebunden }\end{array}$ & intentional & intentional \\
\hline Kontrollvariable & positiv & negativ & positiv & negativ \\
\hline $\begin{array}{l}\text { Zeichen des signifi- } \\
\text { kanten Symbols }\end{array}$ & Geldsumme & Befeht & Rat & Ermahnung \\
\hline generalisierter Wert & Nutzen & $\begin{array}{c}\text { Effektivität der } \\
\text { Zielerreichung } \\
\text { (Legalität) }\end{array}$ & Loyalität & Integrität \\
\hline realer Wert & $\begin{array}{l}\text { individueller } \\
\text { Nutzen }\end{array}$ & $\begin{array}{l}\text { Rechtssicherheit } \\
\text { durch Gehorsam }\end{array}$ & $\begin{array}{c}\text { Schaffung oder } \\
\text { Erneuerung von } \\
\text { Gemeinschaft }\end{array}$ & $\begin{array}{l}\text { Pflichterfül- } \\
\text { lung }\end{array}$ \\
\hline Deckung & $\begin{array}{c}\text { Stabilität der } \\
\text { allgemeinen } \\
\text { Geldwirtschaft }\end{array}$ & Zwangsmittel & $\begin{array}{l}\text { Vertrauen in } \\
\text { Träger von } \\
\text { Prestige }\end{array}$ & Gewissensbiss \\
\hline $\begin{array}{l}\text { institutioneller } \\
\text { Code }\end{array}$ & Privatrecht & $\begin{array}{l}\text { Autorisierung } \\
\text { zur Norm- } \\
\text { erzeugung }\end{array}$ & Prestigeordnung & $\begin{array}{l}\text { moralische } \\
\text { Führung }\end{array}$ \\
\hline $\begin{array}{l}\text { Zusammenführung } \\
\text { von Deckung und } \\
\text { generalisiertem } \\
\text { Wert }\end{array}$ & Machtmedium & $\begin{array}{l}\text { rechtliches } \\
\text { Wissen }\end{array}$ & $\begin{array}{c}\text { Wahrheit/Rich- } \\
\text { tigkeit (insofern } \\
\text { kein medialer } \\
\text { Charakter) }\end{array}$ & $\begin{array}{l}\text { traditionale } \\
\text { Moral }\end{array}$ \\
\hline $\begin{array}{l}\text { Legitimation des } \\
\text { Mediengebrauchs } \\
\text { reflektiert in }\end{array}$ & $\begin{array}{l}\text { Theorie der } \\
\text { politischen } \\
\text { Ökonomie }\end{array}$ & $\begin{array}{c}\text { Philosophie des } \\
\text { Rechts }\end{array}$ & $\begin{array}{c}\text { Wissenschafts- } \\
\text { theorie (insofern } \\
\text { kein medialer } \\
\text { Charakter) }\end{array}$ & Tugendlehre \\
\hline
\end{tabular}

Tab. 2: Übersicht über die Komponenten der Steuerungsmedien

Der Träger des Mediums (Geldsummen) vermag zwischen den Akteuren $\mathrm{zu}$ zirkulieren, in exklusive Verfügungsgewalt genommen, deponiert und wieder investiert zu werden. ${ }^{90}$ Damit der wirtschaftlichen Transak-

90 Siehe MÜNCH, Theorie des Handelns (Fn. 13), 126; HABERMAS, Theorie des kommunikativen Handelns, Bd. 2 (Fn. 7), 397; GIESEN, Die Entdinglichung des Sozialen (Fn. 5), 227. 
tionen zugrunde liegende Code (Eigentum, Vertrag, Zentralbank) seine integrative Funktion erfüllen kann, hat er freilich durch die Operationen des Machtmediums gestützt zu werden. Die Rechtsregeln, die den Gebrauch des Geldes ermöglichen, müssen effektiv durchsetzbar sein. Geld verweist daher auf der Ebene seines eigenen institutionellen Codes auf Macht.

Für das Machtmedium ist die Resymmetrierung nicht einfach durch die Institutionalisierung einer effektiven Herrschaftsordnung durchzuführen. ${ }^{91}$ Am Zusammenspiel der Perspektiven von Deckung ${ }^{92}$ und genereller Wertorientierung tritt dies deutlich hervor. Unmittelbare Deckung wird einem Befehl durch die Verfügung über Zwangsmittel verschafft. Die unterstellte Motivlage beruht hier wie beim Geld auf einem egozentrischen Kalkül, 93 während für den Zusammenhang von generellem und realem Wert auf die effektive Verwirklichung kollektiver Ziele durch einen Akt des individuellen Gehorsams abgestellt wird. ${ }^{94}$ Parsons selbst hat darauf hingewiesen, dass damit die motivierende Kraft des bloßen Nutzenkalküls überschritten wird..$^{95}$ Das wird von Habermas folgendermaßen erläutert:

91 Siehe PARSONS, On the Concept of Influence (Fn. 7), 366-367.

$92 \mathrm{Zu}$ beachten ist freilich generell, dass die Verfügung über Deckungsreserven bei keinem anderen Medium so klaglos funktionieren kann wie beim Geld. Um die Konditionierung überall nach dem Reiz-Reaktionsschema vollziehen zu können, wären einige ausschließlich dem Geld zukommende Eigenschaften vonnöten. Das Mittel müsste, damit die zweckrationale Orientierung des Empfängers vom Sender angepeilt werden kann, physisch kontrolliert, gemessen, entäußert und deponiert werden können. Außerdem hätte es zirkulierbar zu sein. Letzteres ist für Einfluss und Wertbindung nur sehr eingeschränkt der Fall. Beide sind zu stark an Persönlichkeiten gebunden, als dass man sie weitergeben oder entäußern könnte. Weder lassen sich die ihnen zugeordneten Deckungsreserven (wie der gemeinsame kulturelle Hintergrund oder Schuldgefühle) physisch kontrollieren, noch lassen sie sich messen oder kalkulieren. Aber auch für das Machtmedium zeigen sich hier starke Asymmetrien. Die Zirkulierbarkeit der Macht ist wenigstens äußerst begrenzt, wenn nicht überhaupt ausgeschlossen, da sie an die Rechtsförmigkeit einer Delegation gebunden ist. Ein Befehlsempfänger erhält keine Macht. Durch eine Weisung wird Macht nicht weitergegeben, sondern ausgeübt Auch kann man sie nicht einfach an einen Stellvertreter weiterreichen; die Ausübung von Macht wird damit übertragen, nicht aber die Macht selbst. Auch lässt sich Macht nicht einfach messen. Messbar wird sie nur im Hinblick auf eine monetäre Umrechnung der Konsequenzen der Nichtbefolgung eines Befehls. Insofern verhält sich Macht hier gegenüber dem Geld parasitär. Auch lässt sich Macht nicht einfach deponieren. Die mit einem Amt rechtlich festgelegte Kompetenz ist aber ein unerschöpfliches Depot für die Normsetzung - und kann daher in mengenmäßigen Analogien überhaupt nicht erfasst werden. Siehe hiezu HABERMAS, Theorie des kommunikativen Handelns, Bd. 2 (Fn. 7), 402, 411; GIESEN, Die Entdinglichung des Sozialen (Fn. 5), 232-233.

93 Siehe zu letzterem HABERMAS, Theorie des kommunikativen Handelns, Bd. 2 (Fn. 7), 407.

94 Siehe HABERMAS, Theorie des kommunikativen Handelns, Bd. 2 (Fn. 7), 401-402, 409.

95 Siehe PARSONS, On the Concept of Political Power (Fn. 5), 308-309; DERS., On the Concept of Influence (Fn. 7), 365. Sollte das Ziel den an einer Kooperation beteiligten Ak- 
"Offenbar behält Macht als Medium noch etwas von der Befehlsmacht zurück, die, sprechhandlungstheoretisch ausgedrückt, eher mit normierten Aufforderungen als mit einfachen Imperativen zusammenhängt. Diese Bindung lässt Macht für die Rolle eines Steuerungsmediums, das vom Aufwand und Risiko sprachlicher Konsensbildung entlasten soll, weniger geeignet erscheinen als Geld, das keiner Legitimation bedarf." 96

Damit man sich auf die Interaktion in einem System einlässt, in welchem die Übelsvermeidung eine Ausfallsbürgschaft gegenüber der motivierenden Kraft der Moral übernimmt, hat die Machtausübung rechtmäßig zu sein. Denn das Recht erhebt den Anspruch, die Bedingungen legitimer Zwangsausübung zu bestimmen. Damit also das Zusammenspiel von Deckung und generalisiertem Wert (und somit die Resymmetrierung) funktioniert, hat der institutionelle Code der Macht auf gesichertes rechtliches Wissen zu verweisen. Das Steuerungsmedium, das solcherart auf der Ebene des institutionellen Codes der Macht ins Spiel kommt, ist die juristische Expertise.

Ähnliche Beobachtungen lassen sich auch für andere Medien anstellen. In politischen Kontexten besteht für den Einfluss der reale Wert entweder in der Schaffung und Reproduktion von Gemeinschaften, die auf gemeinsamen Überzeugungen beruhen, oder auch bloß in der Rechtfertigung eines kollektiven Interesses ${ }^{97}$ Auf diese Art erfährt der generalisierte Wert der Loyalität seine Konkretisierung. Bei der Wertbindung verhält es sich analog. Sie betrifft die Einforderung von konkreten moralischen Verpflichtungen zur Erhaltung der moralischen Ordnung. In beiden Fällen geht die Wertperspektive über den egozentrischen Erfolgskalkül hinaus. In gleichsam „reiner Form” könnte Einfluss als Medium nur dann fungieren, wenn man davon ausgehen könnte, dass die Loyalität der Adressaten gegenüber der moralischen Überlieferung den angestrebten individuellen Handlungserfolg darstellt. Schon aus diesen Gründen mag die mediale Qualität von Einfluss und Wertbindung zweifelhaft erscheinen. Zweifel ergeben sich aber auch hinsichtlich der Deckung.

Seine Deckung erhält der Einfluss nach Parsons aus dem Vertrauen, das einer angesehenen Person entgegengebracht wird. ${ }^{98}$ Für die Wert-

teur empirisch motivieren, wäre nicht mehr klar, weshalb der Zwang eine Deckungsreserve darstellen müsste.

${ }^{96}$ HABERMAS, Theorie des kommunikativen Handelns, Bd. 2 (Fn. 7), 406.

97 Siehe PARSONS, On the Concept of Influence (Fn. 7), 356, 370; DERS., Social Structure and the Symbolic Media of Interchange (Fn. 37), 102-104.

98 Siehe PARSONS, On the Concept of Influence (Fn. 7), 368; 369: „A unit wields influence in proportion as, in the relevant context, its unverified declarations of information and intention are believed to be responsibly made. This is the 'reputational' parallel to financial credit standing." 
bindung ist in diesem Zusammenhang an die Schuldgefühle zu denken, die für den Fall der Nichterfüllung einer Pflicht zu entstehen drohen. ${ }^{99}$ Während sich bei letzterer das Zusammenspiel von Wertorientierung und Deckung als Ausfluss eines konventionellen Moralverständnisses deuten ließe, werden Akteure sich auf die genannte Abstützung von Einfluss nur einlassen, wenn die Institutionalisierung der Reputation der betreffenden Personen eine bestimmte Qualität aufweist. ${ }^{100}$ Einfluss erwirbt eine Person durch den (etwa als "wissenschaftlich" oder „erfahren”) ausgezeichneten Nimbus von Stellungnahmen, die von einer Position aus abgegeben werden, von deren Inhabern man annimmt, dass sie über begründetes Wissens verfügen. ${ }^{101}$ Aussagen gelten eben als wahr oder richtig, weil der Träger von Einfluss es behauptet.

„,Einfluss' zehrt von der Ressource der Verständigung, aber er stützt sich auf einen Vorschuss an entgegengebrachtem Vertrauen in aktuell nicht überprüfte Überzeugungsmöglichkeiten." ${ }^{102}$

Durch das Vertrauen in die einflussreiche Person werden Begründungsreserven in den kulturellen und sozialisatorischen Hintergrund verschoben. ${ }^{103}$ Vom Experten wird eben vermutet, dass er weiß, was er sagt. Da aber die sachliche Richtigkeit des Gebrauchs des „Reputationscodes"104 sich nicht erweisen lässt, ohne die sachliche Richtigkeit von Aussagen selbst zu überprüfen, kann Einfluss nicht dauerhaft als Me-

\footnotetext{
${ }^{99}$ Da damit ausgeschlossen sei, dass es sich bei diesen Formen der Kommunikation wegen der mangelnden Orientierung am individuellen Handlungserfolg einerseits und der verbleibenden Einbettung in die Lebenswelt andererseits um Medien handeln könne, begreift Habermas beide als "generalisierte Formen der Kommunikation", die den Effekt, vom Dissensrisiko und den interpretativen Aufwand verständigungsorientierten Handelns zu entlasten, auf anderem Wege erreichen. Die Signale, die von Geld und Macht ausgehen, lenken die Interaktion über Eingriff in die Handlungssituation des Adressaten. Das ist bei Einfluss und Wertbildung nicht der Fall. Sie bleiben, laut Habermas, auf die „(...) Bindungseffekte des verständigungsorientierten Handelns angewiesen". Bei an sich intakter Verständigungsorientierung erreicht man also mit ihnen den entlastenden Effekt durch eine besondere Form der "Deckung". Sie basiert auf dem Autoritätsgefälle zwischen Sender und Empfänger, aufgrund dessen der „Mechanismus der Verständigung" auf einer "höheren Stufe" institutionalisiert wird (siehe HABERMAS, Theorie des kommunikativen Handelns, Bd. 2 (Fn. 7), 412, 417).

100 Siehe zur "Technisierung der Lebenswelt" HABERMAS, Theorie des kommunikativen Handelns, Bd. 2 (Fn. 7), 394: „Die Umstellung der Handlungskoordinierung von Sprache auf Steuerungsmedien bedeutet eine Abkoppelung der Interaktion von lebensweltlichen Kontexten überhaupt." Dadurch werde der Freiheitsgrad erfolgsorientierten Handelns erweitert.

101 Siehe HABERMAS, Theorie des kommunikativen Handelns, Bd. 2 (Fn. 7), 412.

102 HABERMAS, Faktizität und Geltung (Fn. 7), 439.

103 Siehe HABERMAS, Theorie des kommunikativen Handelns, Bd. 2 (Fn. 7), 412.

104 Siehe LUHMANN, Die Wissenschaft der Gesellschaft (Fn. 20), 246-251.
} 
dium institutionalisiert werden. ${ }^{105}$ Die Modalität der Deckung gestattet es, Autoritäten zu hinterfragen. ${ }^{106}$ Nur durch den Aufschub des Begründens ist der Einfluss vom verständigungsorientierten Handeln verschieden. Dadurch ist nicht ausgeschlossen, dass er als „unselbstständiges" Medium fungiert, sobald auf Deckungen zurückgegriffen wird, die auch bei Geld und Macht anzutreffen sind. Dies ist angesichts der Tatsache, dass sich die für den Adressaten vorausgesetzte Orientierung an Loyalität gleichfalls nicht generalisieren lässt, durchaus plausibel. ${ }^{107}$ Der Meinung einer "Autorität" folgt man dann eben aus einem egozentrischen Nutzenkalkül, um Nachteile von sich abzuwenden.

Koppelungs- und Substitutionseffekte dieser Art werden uns nun bei der Diskussion des Mediums rechtlichen Wissens weiter beschäftigen.

\section{Idealform und Konvertierung der juristischen Expertise}

Für Parsons dürfte es offensichtlich gewesen sein, dass die juristische Expertise als Unterfall des Einflusses zu betrachten ist. ${ }^{108}$ Wie bei anderen Normensystemen auch werde hier die verbindliche Auslegung von Normen in die Hände von "Experten" gelegt. Das erfahre in derjenigen Phase der Rechtsfindung seine Bestätigung, in welcher die richtige Interpretation und Anwendung von Rechtsregeln zum zentralen Bezugspunkt eines Verfahrens werde: „Here the prototype is the process of interpretation of legal norms in the appellate phase of the judicial process." 109

Die juristische Expertise signalisiert die Aktualisierung fachlicher Kompetenz. Darin liegt gewiss die Ähnlichkeit mit der Ausübung von Einfluss als Mittel der Überredung. Wollte man die Analogie weiterspinnen, dann fände die juristische Expertise ihre Deckung im Vertrauen auf das Wort des Rechtswissenschaftlers, dessen Gewicht („Wert”)

\footnotetext{
${ }^{105}$ HABERMAS, Theorie des kommunikativen Handelns, Bd. 2 (Fn. 7), 412, bezeichnet ihn daher als eine Form generalisierter Kommunikation, die im Gegensatz zu Medien keine besondere institutionelle Rückkoppelung erfordert.

${ }^{106}$ Man mag einwenden, dass Ähnliches doch auch für die Macht der Fall sei, wenn die Verfügung über Zwangsmittel auf die Probe gestellt wird. Ein "machtloser Machthaber" könne doch die Realisierung kollektiver Ziele nicht mehr gewährleisten. Indes wäre durch die Entwaffnung die für die Legitimation des Mediengebrauchs erforderliche Integration der Perspektiven von Deckung und generalisiertem Wert nur mittelbar betroffen. Eine Niederlage macht einen Herrscher noch nicht illegitim, wohl aber den Wissenschaftler der Nachweis der Scharlatanerie.

107 Siehe HABERMAS, Theorie des kommunikativen Handelns, Bd. 2 (Fn. 7), 417-418.

108 Siehe PARSONS, On the Concept of Influence (Fn. 7), 377-378.

109 PARSONS, On the Concept of Influence (Fn. 7), 377.
} 
vom Prestige des jeweiligen Fachmanns abhinge. Da der generalisierte Wert der juristischen Expertise darin bestünde, dass sich die Normadressaten an dem orientieren, was geltendes Recht ist, würde die institutionalisierte juristische Selbstreflexion (die "interpretative Praxis") ${ }^{110}$ als Code das Zusammenspiel von geglaubtem Wissen (Deckung) und der Anwendung dessen, was Recht ist (Wertorientierung), gewährleisten. Rechtliches Wissen existierte im Verhältnis zu Normadressaten, indem es verwertbar würde, stets auch als einlösbares Versprechen, das positive Recht darzustellen. Das einzige Problem, das diese konsequent durchgeführte Analogie aufwirft (siehe Tab. 3), besteht darin, dass rechtliches Wissen in dieser Form wohl nicht (mehr) existiert. ${ }^{111}$ Die Zusammenführung von Deckung und generalisiertem Wert wird nicht (mehr) durch eine interpretative Praxis gesteuert.

Die bei Parsons selbst angesprochene Einbettung der juristischen Expertise in Verfahren, in denen kollektiv bindende, mit Zwangsmitteln bewehrte Entscheidungen getroffen werden, gibt zu erkennen, dass sich die juristische Expertise nicht umstandslos einem Medium zuordnen lässt, das als Mittel der Überredung positiv auf die Überzeugungen anderer wirken soll. ${ }^{112}$ Die Tatsache, dass die in einer juristischen Expertise verwendeten Argumente an allgemein Gültiges appellieren, lässt sie der Ausübung von Einfluss zwar analog erscheinen;113 allerdings kommt damit bloß das komplizierte Verhältnis von Deckung, Wertorientierung und institutionellem Code zum Ausdruck, das für eine Form der Interaktion charakteristisch ist, die sich durch Konvertierungen und Koppelungen mit anderen Medien auszeichnet.

Das kann man zunächst anhand der Legitimation des Mediengebrauchs im Verhältnis zu realen Deckungsverhältnissen erschließen.

110 Siehe im Anschluss an Dworkin bei ALEXANDER SOMEK, Rechtssystem und Republik. Über die politische Funktion des systematischen Rechtsdenkens. Wien 1992, 222-228.

111 Im Gegenteil scheint diese Analogie ideale Anforderungen wiederzugeben, welche die Rechtswissenschaft an sich selbst richtet, da es das Verhältnis von Dogma und wissenschaftlicher Reflexion im Wege der Auszeichnung von Autoritäten widerspiegelt.

112 Siehe PARSONS, On the Concept of Influence (Fn. 7), 356; DERS., Social Structure and the Symbolic Media of Interchange (Fn. 37), 102.

113 Siehe PARSONS, On the Concept of Influence (Fn. 7), 363: „... [P]ersuasion is ego's at tempt to get compliance by offering reasons why it would, from alter's own point of view, independent of situational advantages, 'be a good thing' for him to act as ego wished." 


\begin{tabular}{c|c|c|c|c} 
& $\begin{array}{c}\text { Zeichen des } \\
\text { signifikanten } \\
\text { Symbols }\end{array}$ & $\begin{array}{c}\text { Steuerungs- } \\
\text { variable }\end{array}$ & $\begin{array}{c}\text { Kontroll- } \\
\text { variable }\end{array}$ & generalisierter Wert \\
\hline $\begin{array}{c}\text { Rechtliches } \\
\text { Wissen in } \\
\text { Analogie zu } \\
\text { Einfluss }\end{array}$ & $\begin{array}{c}\text { Juristische } \\
\text { Fachsprache in } \\
\text { einer Aussage } \\
\text { über vorge- } \\
\text { schriebenes } \\
\text { Verhaiten }\end{array}$ & intentional & positiv & $\begin{array}{c}\text { Orientierung am } \\
\text { geltenden Recht }\end{array}$ \\
\hline $\begin{array}{c}\text { (Fortsetzung) } \\
\text { realer Wert }\end{array}$ & Deckung & $\begin{array}{c}\text { institutio- } \\
\text { neller Code }\end{array}$ & $\begin{array}{c}\text { Zusammenführung } \\
\text { von Deckung und } \\
\text { generalisiertem Wert }\end{array}$ \\
\hline $\begin{array}{c}\text { Analogie zu } \\
\text { Einfluss }\end{array}$ & $\begin{array}{c}\text { Bestimmung } \\
\text { eines Rechts- } \\
\text { anspruchs }\end{array}$ & $\begin{array}{c}\text { Vertrauen in } \\
\text { die gutach- } \\
\text { tende Fach- } \\
\text { person }\end{array}$ & $\begin{array}{c}\text { Interpreta- } \\
\text { tive Praxis } \\
\text { rechtlichen } \\
\text { Wissens }\end{array}$ & $\begin{array}{c}\text { Juristische } \\
\text { Selbstreflexion }\end{array}$ \\
\hline
\end{tabular}

Tab. 3: Die juristische Expertise in Analogie zum Einfluss (bzw. als "Abbild" der interpretativen Praxis)

Akzeptabel ist der allgemeine Gebrauch der juristischen Expertise deshalb, weil es mehr als unzweckmäßig wäre, die Rechtsfindung und Rechtsprechung den Normadressaten selbst zu überlassen. Um sicherzustellen, dass sich die Adressaten am geltenden Recht orientieren, ist es günstiger, wenn sie ihr Verhalten mit Blick auf die geschulte juristische Expertise einrichten. Die „organische List" ${ }^{\prime 114}$ des Mediums besteht reell darin, die Motivation, wie vorgeschlagen zu handeln, in Kontexten zweckrationalen (erfolgsorientierten) Handelns zu verankern. Die Rechtmäßigkeit des Verhaltens wird gerade dann gewährleistet, wenn die Normadressaten alles andere bezwecken, als normenkonform zu handeln. ${ }^{115}$

Die im Medium antizipierte Verknüpfung ${ }^{116}$ von Interaktionsangebot und Annahmemotiv kann nur dann dauerhaft erfolgreich vollzogen

\footnotetext{
114 Siehe dazu oben S. 413.

115 Ähnlich sichert des Medium Macht die Festlegung und Verwirklichung kollektiver Ziele im Gewand der individuellen Folgenorientierung. Siehe näher bei HABERMAS, Theorie des kommunikativen Handelns, Bd. 2 (Fn. 7), 405-406.

116 Insofern handelt es sich bei Interaktionsmedien um signifikante Symbole. Siehe MEAD, Eine behavioristische Erklärung des signifikanten Symbols (Fn. 47), 296-297.
} 
werden, wenn die Verwendung des Mediums einerseits im Hinblick auf individuelle Motive hinreichend abgedeckt und andererseits aufgrund vernünftiger Gründe generell akzeptabel ist. Ersteres ergibt sich für die juristische Expertise daraus, dass die Nichtbefolgung eines fachlichen Rats zu Nachteilen führt (da man letztlich mit der Rechtsordnung in Konflikt gerät); ${ }^{117}$ letzteres wird durch die Überlegung nahe gelegt, dass es ratsam ist, die Vermittlung rechtlichen Wissens einem Berufsstand zu übertragen, der kraft der Sozialisation seiner Mitglieder und der wissenschaftlich verbürgten Selbstkontrolle zu garantieren vermag, dass im Einzelfall das geltende Recht zur Anwendung gelangt. Der legitime Mediumgebrauch muss also voraussetzen, dass das juristische Argument in der Lage ist, das positive Recht darzustellen. Es muss möglich sein, eine Reserve an Argumenten aufzubieten, die ein solches Repräsentationsverhältnis begründen. ${ }^{118}$ Als Steuerungsmedium bleibt die juristische Expertise daher auf die Rekonstruktion der theoretischen Grundlagen der Rechtserkenntnis angewiesen.

Gleichwohl ist nicht zu übersehen, dass der juristischen Expertise, was ihre Absicherung in Kontexten erfolgsorientierten Handelns angeht, eine prekäre Unselbständigkeit eigentümlich ist. Der Hinweis auf die prestigeträchtige hierarchia doctorum allein würde nicht ausreichen, bei den Adressaten jenes erfolgsorientierte Handlungsmotiv hervorzubringen, auf welchem die Operation des Mediums zu beruhen hat. ${ }^{119}$ Als Steuerungsmedium ist die juristische Expertise vielmehr in ihrem Verwertungskontext auf die Koppelung mit anderen Medien angewiesen, die zur ihrer eigenen Legitimation wiederum auf die rechtliche Expertise verweisen. Das Geld bedarf, damit seine reziproke Akzeptanz garantiert ist, der Institutionalisierung durch eine vertrauenssichernde Zwangsordnung, während die dazu erforderliche Institutionalisierung von Macht wiederum nur solange legitim sein kann, wie ihre Ausübung rechtmäßig ist. ${ }^{120}$ Die beiden Steuerungsmedien, von denen sich

\footnotetext{
117 Auf die Tatsache, dass dieses Medium daher in sich unvollständig ist, wird noch zurückzukommen sein. Die Absicherung ergibt sich für das Medium Macht aus einem Sanktionspotential und für das Medium Geld durch die Verfügung über hinreichendes Vermögen.

118 Ebenso kann Geld als Medium nur fungieren, solange sein Gebrauch reziprok institutionalisiert und durch Inflationen nicht gefährdet wird, da es ansonsten nicht dazu taugen würde, den Adressaten die erwünschten Güter zu verschaffen. Auch hat Macht einerseits durch Zwangsmittel hinreichende Deckung zu erfahren und andererseits im Rahmen einer Rechtsordnung ausgeübt zu werden, was den konkreten Mediengebrauch erst zu legitimieren vermag. Siehe hierzu PARSONS, Social Structure and the Symbolic Media of Interchange (Fn. 37) 98.

119 Siehe MÜNCH, Recht als Medium der Kommunikation (Fn. 7), 81.

120 Siehe hier nur MACPHERSON, Die politische Theorie des Besitzindividualismus (Fn. 76), 268-294.
} 
die juristische Expertise, um funktionstüchtig zu sein, also abhängig zu machen hat, setzen dieses selbst voraus. Die sich daraus ergebenden Beziehungen zwischen den Steuerungsmedien lassen sich aufklären, indem man darauf achtet, dass das Medium der juristischen Expertise in seinem Verwertungskontext sowohl eine einfache als auch eine doppelt konvertierte Form annimmt. ${ }^{121}$ Daran wird auch erkennbar, dass die Medien Geld, Macht und juristische Expertise in einem wechselseitigen Bedingungsverhältnis stehen.

\section{Einfache und doppelte Konvertierung der juristischen Expertise}

Es ist ein Gemeinplatz, dass sich mögliche Rechtsfolgen, betrachtet man sie als die vorauszuberechnenden Reaktionen staatlicher Organe, in die Kalkulation vermögenswerter Erfolgsaussichten einbeziehen lassen. Das bedeutet, dass sich machtgesteuerte Interaktionen, welche die Institutionalisierung des Geldmediums gewährleisten, vermittels der juristischen Expertise auf die Operationsweise des Geldmediums rückbeziehen lassen. In der ökonomischen Sphäre zirkuliert das rechtliche Wissen daher in einfach konvertierter Form. ${ }^{122}$ Die juristische Expertise hebt die vom Machtmedium gezogenen Grenzen des Geldes selbst in monetärer Form hervor. Für das Geldmedium tritt die Vermittlung in der Form der Verrechnung von Rechtsfolgen auf. Die Kostenabschätzung legalen Verhaltens entspringt einer Selbstreflexion des Geldes. Da die Reflexivität des Geldes bloß auf die Operationen des Machtmediums abzielt, geht das vermittels des Rekurses auf solche Operationen ins Spiel gebrachte rechtliche Wissen in der allgemeinen Nutzenorientierung auf. Ungeachtet der Tatsache, ob eine Behördenpraxis gemessen an internen Maßstäben rechtmäßig ist, werden bloß deren Kosten oder der von ihr zu erwartende Nutzen abgeschätzt. Die einfache Konvertierung der juristischen Expertise in die Selbstreflexion des Geldes erfasst das rechtliche Wissen vollständig. Das rechtliche Wissen findet sich machtgesteuerten Interaktionen subordiniert. Das ist der Fall, ob-

${ }^{121}$ Zum hier verwendeten Begriff der "Konversion“ siehe ANTHONY GIDDENS, The Constitution of Society. Outline of a Theory of Structuration. Oxford 1984, 301-302. Der Unterschied zu Parsons' Vorstellung von einem "Austausch“ der Medien besteht darin dass Steuerungsmedien durch die Koppelung von Elementen unter dem Gesichtspunkt der Deckung eine andere Qualität erhalten; vgl. PARSONS, On the Concept of Influence (Fn. 7), 371-372.

122 Das Beispiel des „Freikaufens" von der Verpflichtung, einen bestimmten Anteil Behinderter einzustellen, zeigt, dass rechtliche Regelungen selbst diese Konversionsform von Recht in sich aufnehmen. 
wohl die Legitimität der Ausübung von Macht an sich auf Bedingungen beruht, die durch das Recht gezogen werden (siehe Tab. 4).

\begin{tabular}{c|c|c|c|c} 
& $\begin{array}{c}\text { Zusammen- } \\
\text { führen von } \\
\text { Deckung und } \\
\text { generalisiertem } \\
\text { Wert }\end{array}$ & $\begin{array}{c}\text { Selbstreflexion } \\
\text { des Mediums }\end{array}$ & Koppelung & $\begin{array}{c}\text { Folge für juristische } \\
\text { Expertise }\end{array}$ \\
\hline $\begin{array}{c}\text { Einfache } \\
\text { Konvertie- } \\
\text { rung (Geld) }\end{array}$ & Machtmedium & $\begin{array}{c}\text { Verrechnung } \\
\text { von vorherseh- } \\
\text { baren Rechts- } \\
\text { folgen }\end{array}$ & $\begin{array}{c}\text { Orientierung } \\
\text { am Recht und } \\
\text { Nutzen }\end{array}$ & $\begin{array}{c}\text { Substitution des gene- } \\
\text { ralisierten Wertes der } \\
\text { Rechtsmäßigkeit } \\
\text { durch Nützlichkeit }\end{array}$
\end{tabular}

Tab. 4: Einfache Konvertierung der juristischen Expertise

Einschätzungen des jeweiligen wirtschaftlichen Risikos beeinflussen individuelle Entscheidung darüber, gegen eine bestehende Praxis der Rechtsanwendung in einem Verfahren anzukämpfen. Die einfache Konvertierung geht in diesem Fall in die doppelte Konvertierung über. Sie wird in Koppelung mit dem Machtmedium vollzogen. Hier stößt man auf den Angelpunkt für die allgemein akzeptable Deckung der juristischen Expertise durch egozentrische Erfolgskalküle. ${ }^{23}$ Sie wird dadurch bewirkt, dass Rechtssubjekte durch die Androhung von Sanktionen dazu angehalten werden, sich befehlsgemäß zu verhalten. Was aus der Sicht der idealen Version der juristischen Expertise sich (wegen der Subordination des Machtmediums unter diese) als Verrechtlichung von Herrschaft darstellen sollte, ${ }^{124}$ verkehrt sich in Vermachtung rechtlichen Wissens. Zwar entspricht die Forderung, die Ausübung von Herrschaft habe dem Recht zu genügen, einer Selbstreflexion von Macht, welcher der juristischen Expertise eine regulierende Funktion zubilligt; allerdings geht die juristische Expertise in ihrem Verwertungskontext in der Selbstreflexion der Macht auf. Das geschieht nicht zufällig. Es ist in dem Problem begründet, das rechtliches Wissen dazu nötigt, eine mediale Gestalt anzunehmen. Das Recht muss verbindlich gewusst werden. Deswegen hat die Institutionalisierung der medialen Form rechtli-

${ }^{123}$ Siehe bloß JOHANN GOTTLIEB FICHTE, Grundlage des Naturrechts nach Prinzipien der Wissenschaftslehre (1794). 3. Aufl., Hamburg 1979, 201-202; FRIEDRICH WILHELM JOSEPH VON SCHELIING, System des transzendentalen Idealismus (1800). Hamburg 1957, 251.

${ }^{124}$ Siehe KLAUS EDER, Geschichte als Lernprozess? Zur Pathogenese politischer Modernität in Deutschland. Frankfurt am Main 1985, 94-97. 
chen Wissens ${ }^{125}$ auf die Selbstreflexion des Machtmediums zugeschnitten zu sein. ${ }^{126}$ So wie der leitende Gesichtspunkt der Selbstreflexion des Geldes in der Abschätzung der Kosten rechtmäßigen Verhaltens besteht, wird aus der Perspektive einer Selbstreflexion der Macht das geltende Recht im Spiegel der effektiven Fortsetzung organisationsspezifischer Aufgaben relevant. Zwar funktioniert beim Machtmedium, was die Normadressaten angeht, die Konversion der offiziellen juristischen Expertise in die Abschätzung von Handlungsfolgen genauso wie beim Geldmedium, denn auch hier sind egozentrische Erfolgskalküle im Spiel; allerdings besteht, was die Folgen der Konversion angeht, auch ein nicht zu vernachlässigender Unterschied. Institutionalisierte Interpretenmacht ermöglicht es, einen Stil der juristischen Argumentation auf Dauer zu stellen. ${ }^{127}$ Sobald es aber Instanzen gibt, die einen bestimmten Begründungsstil vorgeben, ist implizit auch darüber entschieden, was in der juristischen Argumentation überhaupt relevant zu werden vermag.

An der strategischen Öffnung und Schließung des Umfangs dessen, was als relevant erachtet wird, erkennt man die interne Vermachtung rechtlichen Wissens. Sie betrifft nicht eine augenfällig missbräuchliche Verwendung der juristischen Begründung zur nachträglichen Rationalisierung politisch induzierter Maßnahmen. ${ }^{128}$ Sie bezeichnet vielmehr die selektiven Relevanzschwellen, die von entscheidenden Stellen für die juristische Selbstreflexion errichtet werden. Ein intern vermachtetes rechtliches Wissen nimmt gewisse Argumente nicht wahr. Sie werden als "theoretisch" abgetan. Das bedeutet, dass sie zwar als Bestandteil der juristischen Selbstreflexion anerkannt, aber bloß als nicht "passend" erachtet werden. Ihnen nachzugehen, würde die bestehende Entscheidungspraxis verunsichern.

${ }^{125} \mathrm{MüNCH}$, Recht als Medium der Kommunikation (Fn. 7), 66-67, 71, spricht in diesem Zusammenhang etwas ungeschickt von einem "Zerbrechen der Einheit von Recht und Wirklichkeit", weist aber zurecht darauf hin, dass durch die symbolische Generalisierung (rechtlichen Wissens) für die Normadressaten zunächst offen bleibt, wie das vorhandene Rechtsmaterial im Einzelfall angewendet wird, da sich dies nur durch ein juristisches Gutachten bestimmen lässt.

126 Siehe IMMANUEL KANT, Metaphysische Anfangsgrïnde der Rechtslehre (1797). Herausgegeben von BERND LUDWIG. Hamburg 1986, 126.

127 Das Mysterium der Rechtskraft und die Trägheit ständiger Rechtsprechung vermögen die staatliche Entscheidungstätigkeit auch dann in verbindliches Recht zu verwandeln, wenn die Akademie mit literarischem Nachdruck protestiert. Auch ist das Maß des juristisch nicht Unvertretbaren großzügig dimensioniert. Den Tatbeständen des Asylgesetzes kann eben ein engerer oder ein weiterer Verstand beigelegt werden. Umgehungen des Ablöseverbots können als solche identifiziert oder aber als außerhalb des Normbereichs fallend ausgegeben werden.

128 Siehe dazu Fn. 7 
Die juristische Expertise wird solcherart in die rekursive Schließung des Machtmediums einbezogen. Im Vergleich zur Koppelung mit dem Geldmedium ist der Konvertierungseffekt von anderer Qualität. Während sich in der einfachen Konvertierung das juristische Argument darauf reduziert, die Tätigkeit staatlichen Verhaltens berechenbar zu machen, basiert die doppelte Konvertierung darauf, in konkreten interpretativen Praktiken rechtlichen Wissens Schranken für die Selbstreflexion $z u$ errichten. Durch diese Schranken kann die Organtätigkeit erst jenes nicht mehr zu ignorierende Faktum werden, welches manche Rechtspositivisten zur Erklärung der Rechtsgeltung heranziehen. ${ }^{129}$ Während die einfache Konvertierung die Selbstreflexion rechtlichen Wissens bloß aus der Domäne der Kalkulation wirtschaftlich relevanter Rechtsfolgen hinausschiebt, fällt sie bei doppelter Konvertierung aus dem Bereich heraus, in dem sie eigentlich wirksam werden soll (siehe Tab. 5). ${ }^{130}$ Die juristische Expertise ist nicht mehr durch juristische Argumente gesteuert.

\begin{tabular}{|c|c|c|c|c|}
\hline & $\begin{array}{c}\text { Zusammen- } \\
\text { führen von } \\
\text { Deckung und } \\
\text { generalisiertem } \\
\text { Wert }\end{array}$ & $\begin{array}{l}\text { Selbstreflexion } \\
\text { des Mediums }\end{array}$ & Koppelung & $\begin{array}{l}\text { Folge für die } \\
\text { juristische } \\
\text { Expertise }\end{array}$ \\
\hline $\begin{array}{l}\text { Doppelte } \\
\text { Konver- } \\
\text { tierung }\end{array}$ & $\begin{array}{l}\text { rechtliches } \\
\text { Wissen }\end{array}$ & $\begin{array}{l}\text { Rechtmäßigkeit } \\
\text { der effektiven } \\
\text { Erledigung }\end{array}$ & $\begin{array}{c}\text { Effektive } \\
\text { Verwirklichung } \\
\text { kollektiver Ziele } \\
\text { und Orientierung } \\
\text { an dem, was } \\
\text { Recht ist }\end{array}$ & $\begin{array}{l}\text { Begrenzung der } \\
\text { juristischen Selbst- } \\
\text { reflexion (interne } \\
\text { Vermachtung) }\end{array}$ \\
\hline
\end{tabular}

Tab. 5: Doppelte Konvertierung der juristischen Expertise

129 Siehe Herbert Lionel Adolphus Hart, The Concept of Law. Oxford 1961, 110-114; JOSEPH RAZ, Practical Reason and Norms. 2. Aufl., Princeton 1990, 134-136.

130 Die einfache Konvertierung funktioniert freilich nur dann, wenn die doppelte bereits vollzogen worden ist. Erst im Kontext der entfalteten Staatstätigkeit vermag das Recht ein Bestimmtes $z u$ sein, während die doppelte Konvertierung voraussetzt, dass das Medium Macht rechtmäßig ausgeübt wird. Und dazu reicht es - unter anderem - hin die juristische Selbstreflexion auf jenes Maß zurückzuschneiden, das mit der erfolgreichen Aufgabenerfüllung verbunden ist. Das gilt auch für die Tätigkeit von Gerichten, deren Aufgabe ja darin besteht, eine bindende Entscheidung zu treffen, die mit der bisherigen Rechtsprechung kohärent ist. Siehe hierzu MEIR DAN-COHEN, Law, Community, and Communication, in: Duke Law Journal 1989, 1654-1675, hier 1672. 


\section{Das verlorene Fundament}

Es ist bereits darauf hingewiesen worden, dass die Modellierung der juristischen Expertise nach dem Muster des Einflusses auf einer Idealisierung beruht. Sie gibt zwar eine Anforderung wieder, die das rechtliche Wissen an sich selbst richtet, ihr kommt aber für das Verhältnis zu den Normadressaten keine Wirklichkeit zu. Um die Orientierung der Adressaten am geltenden Recht $\mathrm{zu}$ gewährleisten, ist das rechtliche Wissen notwendig auf die Koppelung mit den Medien Geld und Macht angewiesen. Dadurch kann es an den Deckungen partizipieren, mit denen diese Medien die Annahme von Interaktionsangeboten steuern. Umgekehrt zeitigt diese Partizipation Folgen für die Realisierung des institutionellen Codes dieser Medien. Wegen der in ihnen indirekt (Geld) oder direkt (Macht) vorausgesetzten Abhängigkeit von der juristischen Expertise und wegen der Abhängigkeit, in welche umgekehrt diese gerät, ist die Bestimmung des institutionellen Codes von Geld und Macht sozial unabhängig von der juristischen Selbstreflexion. Für das rechtliche Wissen bedeutet dies, dass es sein Versprechen, das geltende Recht darzustellen, nur durch die Selbstreflexion anderer Medien gebrochen erfüllen kann (siehe Tab. 6). Um es paradox auszudrücken: das Recht kann nur in einer Gesellschaft ohne Recht gewusst werden. Durch das Aufbieten von Theorie lässt sich die Selbststeuerungsfähigkeit rechtlichen Wissens nicht wiederherstellen. Sobald auf der theoretischen Ebene ohnedies alles umstritten ist, ist der Mediengebrauch umso nachhaltiger erforderlich. ${ }^{131}$ Dieser Befund mag zur Schlussfolgerung verleiten, dass die selbstreflexive Wertbestimmung des rechtlichen Wissens einfach durch ökonomische und politische Interessen überformt wird. ${ }^{132}$ Das mag der Fall sein. Doch sozial darf es nicht der Fall sein. Die juristische Expertise darf sich wegen der wechselseitigen Konstitution der Medien nicht einfach darauf einlassen, ihre Rechtfertigung darin zu finden, Verwertungsinteressen mit beliebigen normati-

131 Siehe zu dieser Funktion der Steuerungsmedien HABERMAS, Theorie des kommunikativen Handelns, Bd. 2 (Fn. 7), 393, 408; DERS., Theorie des kommunikativen Handelns, Bd. 1 (Fn. 23), 128, 150; LUHMANN, Einführende Bemerkungen zu einer Theorie der symbolisch generalisierten Steuerungsmedien (Fn. 13), 174.

132 Dementsprechend wird der Gebrauch juristischer Argumente zum Strategiespiel. DAN-COHEN, Law, Community, and Communication (Fn. 130), 1666-1667, 1673, meint dies sowohl für die Argumentationen von Rechtsanwälten wie von Richtern feststellen zu können, wenngleich die Adressaten in den genannten Fällen unterschiedlich seien. Während Rechtsanwälte im Interesse ihrer Klienten letztlich um die Zustimmung des Gerichts konkurrierten, stünden Richter vor der schwierigen Aufgabe, eine Vielzahl von ,audiences" adressieren zu müssen, vor denen die Entscheidungsbegründung eine Frieden und Respekt bewahrende Aufgabe zu erfüllen habe. 
ven Gründen zu versorgen. Auch darf Macht den Gebrauch der juristischen Expertise nicht auf die Dauer verdrängen oder okkupieren. Sie würde inflationären Tendenzen erliegen, zumal sie aufgrund ihres institutionellen Codes von der Integrität der juristischen Expertise abhängig ist. ${ }^{133}$ Wie kann es der juristischen Expertise also gelingen, dennoch gesellschaftliches Ansehen zu haben?

Die beiden nun folgenden Versionen, diesen erklärungsbedürftigen Umstand aufzuhellen und damit dem Zusammenführen von Deckung und generalisiertem Wert - also dem Zusammenspiel zwischen dem Vertrauen in die Expertise und der Anwendung dessen, was Recht ist Plausibilität $\mathrm{zu}$ verleihen, lassen sich aus dem Versuch verstehen, ein mediales Äquivalent der idealen Version der juristischen Expertise in dessen Verwertungskontext zu realisieren.

\begin{tabular}{|c|c|c|c|c|}
\hline & Geld & Macht & $\begin{array}{c}\text { Idealisierte } \\
\text { Version der } \\
\text { juristischen } \\
\text { Expertise }\end{array}$ & $\begin{array}{l}\text { Doppelt konvertierte } \\
\text { juristische Expertise }\end{array}$ \\
\hline $\begin{array}{l}\text { Steuerungsva- } \\
\text { riable }\end{array}$ & $\begin{array}{l}\text { situations- } \\
\text { gebunden }\end{array}$ & $\begin{array}{l}\text { situations- } \\
\text { gebunden }\end{array}$ & intentional & situationsgebunden \\
\hline $\begin{array}{l}\text { Kontrollva- } \\
\text { riable }\end{array}$ & positiv & negativ & positiv & negativ bzw. positiv \\
\hline $\begin{array}{c}\text { Zeichen des sig- } \\
\text { nifikanten } \\
\text { Symbols }\end{array}$ & $\begin{array}{l}\text { Geld- } \\
\text { summe }\end{array}$ & Befehl & $\begin{array}{l}\text { Expertise, } \\
\text { die eine } \\
\text { Aussage } \\
\text { über einen } \\
\text { Rechts- } \\
\text { anspruch } \\
\text { enthält }\end{array}$ & $\begin{array}{l}\text { Expertise, die eine Aussage über } \\
\text { einen Rechtsanspruch enthält }\end{array}$ \\
\hline $\begin{array}{c}\text { generalisierter } \\
\text { Wert }\end{array}$ & Nutzen & $\begin{array}{l}\text { Effektivität } \\
\text { der Zieler- } \\
\text { reichung } \\
\text { (Legalität) }\end{array}$ & $\begin{array}{l}\text { Orientierung } \\
\text { am gelten- } \\
\text { den Recht }\end{array}$ & $\begin{array}{l}\text { a) Koppelung mit Geld: } \\
\text { Kosten rechtmäßigen Verhaltens } \\
\text { b) Koppelung mit Macht: } \\
\text { das in einer Interpreten- } \\
\text { gemeinschaft oder einer } \\
\text { Jurisdiktion Argumentierbare }\end{array}$ \\
\hline
\end{tabular}

133 Siehe dazu schon oben S. 418. 


\begin{tabular}{|c|c|c|c|c|}
\hline & Geld & Macht & $\begin{array}{c}\text { Idealisierte } \\
\text { Version der } \\
\text { juristischen } \\
\text { Expertise }\end{array}$ & $\begin{array}{l}\text { Doppelt konvertierte } \\
\text { juristische Expertise }\end{array}$ \\
\hline realer Wert & $\begin{array}{c}\text { Individu- } \\
\text { eller } \\
\text { Nutzen }\end{array}$ & $\begin{array}{l}\text { Rechts- } \\
\text { sicherheit } \\
\text { durch } \\
\text { Gehorsam }\end{array}$ & \begin{tabular}{|} 
Bestimmung \\
eines \\
Rechts- \\
anspruchs
\end{tabular} & $\begin{array}{c}\text { a) Koppelung mit Geld: } \\
\text { Inkorporation vorhersehbaren } \\
\text { staatlichen Verhaltens in einen } \\
\text { Nutzenkalkül } \\
\text { b) Behauptung im Rahmen } \\
\text { bzw. Fortsetzung einer Praxis } \\
\text { rechtlichen Wissens }\end{array}$ \\
\hline Deckung & $\begin{array}{c}\text { Stabilität } \\
\text { der allge- } \\
\text { meinen } \\
\text { Geldwirt- } \\
\text { schaft }\end{array}$ & $\begin{array}{l}\text { Zwangs- } \\
\text { mittel }\end{array}$ & $\begin{array}{c}\text { Vertrauen in } \\
\text { gutachtende } \\
\text { Fachperson }\end{array}$ & $\begin{array}{l}\text { a) Koppelung mit Geld: Vorher- } \\
\text { sehbarkeit der Entscheidung } \\
\text { b) Redundanz der juristischen } \\
\text { Argumentation }\end{array}$ \\
\hline $\begin{array}{l}\text { institutioneller } \\
\text { Code }\end{array}$ & Privatrecht & $\begin{array}{l}\text { Autorisie- } \\
\text { rung zur } \\
\text { Normer- } \\
\text { zeugung }\end{array}$ & $\begin{array}{l}\text { interpreta- } \\
\text { tive Praxis } \\
\text { rechtlichen } \\
\text { Wissens }\end{array}$ & konkrete Jurisdiktionen \\
\hline $\begin{array}{c}\text { Zusammen- } \\
\text { führen von } \\
\text { Deckung und } \\
\text { generalisiertem } \\
\text { Wert }\end{array}$ & $\begin{array}{l}\text { Macht- } \\
\text { medium }\end{array}$ & $\begin{array}{l}\text { rechtliches } \\
\text { Wissen }\end{array}$ & $\begin{array}{l}\text { Juristische } \\
\text { Selbst- } \\
\text { reflexion }\end{array}$ & $\begin{array}{c}\text { Selbstreflexion der Medien } \\
\text { Macht und Geld: Abstellen auf } \\
\text { Verwertungskontext }\end{array}$ \\
\hline $\begin{array}{l}\text { Selbstreflexion } \\
\text { des Mediums }\end{array}$ & $\begin{array}{l}\text { Verrech- } \\
\text { nung von } \\
\text { vorher- } \\
\text { sehbaren } \\
\text { Rechts- } \\
\text { folgen }\end{array}$ & $\begin{array}{l}\text { Recht- } \\
\text { mäßigkeit } \\
\text { effektiver } \\
\text { Erledigun- } \\
\text { gen }\end{array}$ & $\begin{array}{l}\text { Juristische } \\
\text { Methodik }\end{array}$ & $\begin{array}{l}\text { Brauchbarkeit im Rahmen einer } \\
\text { Interpretengemeinschaft }\end{array}$ \\
\hline $\begin{array}{l}\text { Theoretische } \\
\text { Legitimation des } \\
\text { Medien- } \\
\text { gebrauchs } \\
\text { reflektiert in }\end{array}$ & $\begin{array}{c}\text { Theorie } \\
\text { der politi- } \\
\text { schen } \\
\text { Ökonomie }\end{array}$ & $\begin{array}{l}\text { Philoso- } \\
\text { phie des } \\
\text { Rechts }\end{array}$ & $\begin{array}{c}\text { Rechtstheo- } \\
\text { rie als Selbst- } \\
\text { reflexion } \\
\text { rechtlichen } \\
\text { Wissens (in- } \\
\text { sofern kein } \\
\text { medialer } \\
\text { Charakter) }\end{array}$ & $\begin{array}{l}\text { Verwaltungs- und } \\
\text { Betriebswirtschaftslehre }\end{array}$ \\
\hline
\end{tabular}

Tab. 6: Wechselbestimmung der Medien durch Koppelung und Konversion 


\section{Als wäre es Dialektik der Aufklärung: Der Legalitätsglaube}

Der interne Umsturz des Bedingungsverhältnisses zwischen Recht und Macht, der für das intern vermachtete rechtliche Wissen $\mathrm{zu}$ beobachten ist, lässt sich durch einen gleichsam "magischen" Mediengebrauch auffangen. Die interne Gleichgültigkeit der juristischen Expertise gegenüber ihrer Selbstreflexion erfährt damit eine institutionelle Codierung. Dies wurde erstmals von Max Weber unter dem Titel „Legalitätsglaube ${ }^{\prime \prime 134}$ auf den Begriff gebracht.

Der Legalitätsglaube manifestiert sich nach Weber in der „,... Fügsamkeit gegenüber formal korrekt und in der üblichen Form zustande gekommenen Satzungen". ${ }^{135}$ Seine Indifferenz gegenüber der Begründung der moralischen Gehalte und der Zweckmäßigkeit des geltenden Rechts ist nicht ohne jene innere Ambivalenz, durch die sich die Partizipation der juristischen Expertise an der Selbstreflexion der Medien Geld und Macht kenntlich macht. An den beiden defizienten Modalitäten, solche Satzungen für legitim zu erachten, ${ }^{136}$ hat der Legalitätsglaube daher nicht zufällig Anteil. Vermöge des Legalitätsglaubens werden laut Weber vereinbarte Satzungen, die auf einem Mehrheitsvotum beruhen, auch gegen unterliegende Minderheiten, von Minderheiten oktroyierte Ordnungen aufgrund der normativen Kraft des Faktischen von der Mehrheit als legitim anerkannt. ${ }^{137}$ Diese Akzeptanz hat ihre Grundlage nicht in der Legitimität der Kompromisse ermöglichenden Verfahren ${ }^{138}$ oder in der legitimen Autorität eines Herrschers, sondern allein im Vertrauen auf die Beachtung formalisierter, durch den Verstand nachvollziehbarer ${ }^{139}$ Vorgangsweisen bei der Setzung und Anwendung genereller Normen. Solcherart lässt sich der Schein einer Korrespondenz mit außerprozeduralen Sachverhalten hervorrufen:

134 Siehe MAX WEBER, Wirtschaft und Gesellschaft. Grundriß der verstehenden Soziologie (1921). Herausgegeben von JOHANNES WINCKELMANN, 5. Aufl., Tübingen 1976, 19. Habermas hat natürlich zu Recht darauf hingewiesen, dass der Legalitätsglaube die Legitimität des Rechts nicht ersetzen könne. Siehe HABERMAS, Theorie des kommunikativen Handelns, Bd. 1 (Fn. 23), 361.

135 WEBER, Wirtschaft und Gesellschaft (Fn. 134), 19. Für eine systemtheoretische Fortentwicklung dieser These siehe bekanntlich NIKLAS LUHMANN, Legitimation durch Verfahren. 4. Aufl., Frankfurt am Main 1983, 28-29.

136 Bei nicht-defizienter Modalität handelt es sich um die Vereinbarung aufgrund gemeinsamer Interessen einerseits und den Befehl eines legitimen Machthabers andererseits. Siehe auch WEBER, Wirtschaft und Gesellschaft (Fn. 134), 125.

137 Ebd., 19-20.

138 Siehe dazu HABERMAS, Faktizität und Geltung (Fn. 7), 204-206.

139 Siehe WEBER, Wirtschaft und Gesellschaft (Fn. 134), 396-397. 
„Soweit ,Abstimmungen' als Mittel der Schaffung oder Änderung von Ordnungen legal sind, ist es sehr häufig, dass der Minderheitswille die formale Mehrheit erlangt und die Mehrheit sich fügt: die Majorisierung nur Schein ist." 140

Für die doppelte Konvertierung der juristischen Expertise war festzustellen, dass sich die Perspektiven von Deckung und generalisiertem Wert nicht auf jene Art zusammenführen lassen, welche die idealisierte Version der juristischen Expertise erfordert. Nichts gewährleistet, dass das intern vermachtete rechtliche Wissen wissenschaftlich fundiert ist. Im Vergleich dazu greift beim Legalitätsglauben ein kompensierender Mechanismus ein, der darin besteht, dass die formale Rationalität der Rechtsverfahren von einem gleichsam "magischen” Bewusstsein auf der Seite der Normadressaten und Beteiligten getragen wird. ${ }^{141}$ Dies betrifft den gesellschaftlichen Vorgang der Rechtserkenntnis im Ganzen. Hinsichtlich seiner Geltung beruht das moderne Recht auf Verfahren willkürlicher Rechtssatzung, ${ }^{142}$ deren einzelne Stufen durch juristisches Fachwissen vermittelt werden. ${ }^{143}$ Mit Blick auf den Vollzug einfacher Konvertierung bleibt dabei für den Laien unverständlich, was im Medium der juristischen Begriffsbildung zur Darstellung gelangt. Die ju-

140 WEBER, Wirtschaft und Gesellschaft (Fn. 134), 20.

141 Siehe dazu auch die Andeutungen bei BERNHARD PETERS, Rationalität, Recht und Gesellschaft. Frankfurt am Main 1991, 118.

142 Siehe WEBER, Wirtschaft und Gesellschaft (Fn. 134), 504-505. Zu Webers positivistischem Geltungsverständnis siehe HABERMAS, Theorie des kommunikativen Handelns, Bd. 1 (Fn. 23), 357, 361.

143 Insofern bildet für Weber in der Tat die interne Systematisierung den Kern der Rationalität des modernen Rechts. Siehe WEBER, Wirtschaft und Gesellschaft (Fn. 134), 125130 und dazu HABERMAS, Theorie des kommunikativen Handelns, Bd. 1 (Fn. 23), 348 Die legale Herrschaft ist mit dem ihr zugehörigen Legitimitätstypus des Legalitätsglaubens intern verknüpft, da sie die formale Rationalität des Rechts in die Organisation von Betrieben aufnimmt. Dies wird deutlich bei MAX WEBER, Staatssoziologie. Herausgegeben von JOHANNES WINCKELMANN, 2. Aufl., Berlin 1966, 99-101. Siehe auch GEPHART, Gesellschaftstheorie und Recht (Fn. 6), 576-582. Was das juristische Fachwissen anbelangt, stellen für Weber bekanntlich Generalisierung (die Orientierung an generellen Rechtssätzen) und Systematisierung (Zurückführung der Rechtssätze auf Prinzipien) die Grundbausteine der durch juristische Fachleute vollzogenen begrifflichen Durchdringung des Rechts dar (siehe dazu WOLFGANG SCHLUCHTER, Die Entwicklung des okzidentalen Rationalismus. Eine Analyse von Max Webers Gesellschaftsgeschichte. Tübingen 1979, 133-134). Als Bindemittel für die Herstellung und Reproduktion der systematischen Einheit des Rechtsmaterials dienen die Interpretation von Rechtssätzen und die Konstruktion eines logischen Zusammenhangs zwischen Rechtsbegriffen, der von allen Bezugnahmen auf nicht-formalisierbare (materiale) Wertstandards ethischer Provenienz freigehalten wird. Der solcherart durch die juristische Begriffsarbeit vermittelte Rechtsgang garantiert für Weber Rechtssicherheit im Sinne der "Berechenbarkeit" des Rechts (siehe WEBER, Wirtschaft und Gesellschaft (Fn. 134), 397, 469, 508-509). 
ristische Konstruktion ${ }^{144}$ verhält sich auch gegenüber den Rechtsanschauungen der Laien indifferent:

„Die ,Erwartungen' der Rechtsinteressenten sind an dem ökonomischen oder fast utilitaristischen praktischen ,Sinn' eines Rechtssatzes orientiert; dieser aber ist, rechtslogisch gesehen, irrational. Niemals wird ein ,Laie' verstehen, dass es einen ,Elektrizitätsdiebstahl' bei der alten Definition des Diebstahlsbegriffs nicht geben konnte. Es ist also keineswegs eine spezifische Torheit der modernen Jurisprudenz, welche zu diesen Konflikten führt, sondern in weitem Umfang die ganz unvermeidliche Disparatheit logischer Eigengesetzlichkeiten jedes formalen Rechtsdenkens überhaupt gegenüber den auf ökonomischen Effekt abzweckenden und auf ökonomisch qualifizierte Erwartungen abgestellten Vereinbarungen und rechtlich relevanten Handlungen der Interessenten. ${ }^{\prime 145}$

Der Legalitätsglaube kann demgemäß nicht ausschließlich in einem ökonomischen Kalkül seine Deckung finden. Nach Weber reichen seine Wurzeln bis zum Ursprung der Satzung in der magischen "Rechtsoffenbarung" zurück. ${ }^{146}$ Solche Offenbarung nimmt seines Erachtens von der Tätigkeit von Rechtspropheten ihren Ausgang, um schließlich, in logisch sublimierter Form, in die Kompetenz von Rechtshonoratioren und Fachjuristen überzugehen. ${ }^{147}$ Die Rechtsoffenbarung (als Rechtsweistum oder Orakel eines charismatischen Herrschers oder Priesters) ${ }^{148}$ vermag traditionale Gepflogenheiten von innen heraus zu überschreiten. ${ }^{149}$ In den beiden Formen der daraus hervorgehenden Sat-

144 Die Begriffsjurisprudenz ist ja von Weber zum Paradigma der formalen Rationalität des modernen Rechts erhoben worden. PETERS, Rationalität, Recht und Gesellschaft (Fn. 141), 115

145 WEBER, Wirtschaft und Gesellschaft (Fn. 134), 506.

146 Siehe WEBER, Wirtschaft und Gesellschaft (Fn. 134), 449.

147 "Die formalen Qualitäten des Rechts entwickeln sich dabei aus einer Kombination von magisch bedingtem Formalismus und offenbarungsmäßig bedingter Irrationalität im primitiven Rechtsgang, eventuell über den Umweg theokratisch oder patrimonial bedingter materialer und unformaler Zweckrationalität, zu zunehmend fachmäßig juristischer, also logischer Rationalität und Systematik und damit - zunächst rein äußerlich betrachtet - zu einer zunehmend logischen Sublimierung und deduktiven Strenge des Rechts und einer zunehmend rationalen Technik des Rechtsgangs" (WEBER, Wirtschaft und Gesellschaft (Fn. 134), 504-505).

148 Siehe WEBER, Wirtschaft und Gesellschaft (Fn. 134), 451-452.

149 Die Rechtspropheten stehen mit den magischen oder göttlichen Kräften, die über die Integrität von Traditionen wachen, in mystischem Kontakt. Das legitimiert sie zur Rechtssetzung (ohne dass in diesem Kontext freilich zwischen Anwendung und Satzung begrifflich unterschieden werden könnte, WEBER, Wirtschaft und Gesellschaft (Fn. 134), 450). Auch wird zwischen der Bewahrung der Integrität der geheiligten Tradition und der persönlichen Qualifikation zur Rechtssetzung (als sachliche oder persönliche Legitimation) noch nicht unterschieden. Siehe ebd., 448-449. Notwendig wird sie spätestens dann, "(...) wenn Verschiebungen der ökonomischen und sonstigen Lebensbedingungen neue Normen für bisher nicht geordnete Probleme fordern" (ebd., 446). Sie wird in der Form eines Paktes zwischen Sippen und Stämmen vorgenommen 
zungsbefugnis - das imperium des Herrschers (eines Häuptlings bzw. des Staates) ${ }^{150}$ und die Autorisierung der Rechtsfindung durch eine gelehrte Elite (von Priestern bzw. der juristischen Profession) ${ }^{151}$ - bleibt der irrationale Ursprung in rationalisierter Form erhalten. ${ }^{152}$ Insofern schimmern die archaischen Ursprünge des modernen Rechts im Phänomen des Legalitätsglaubens noch durch. Darauf weist Weber (in unmittelbarem Anschluss an die Erwähnung des Scheins legaler Paktierung) unmissverständlich hin:

„Der Glaube an die Legalität paktierter Ordnungen reicht ziemlich weit zurück und findet sich zuweilen auch bei sog. Naturvölkern: fast stets aber ergänzt durch die Autorität von Orakeln."153

Die Gewöhnung an die legale Herrschaft rührt an vormodernen Legitimitätsmodellen und geht mit ihnen eine verschwiegene Allianz ein:

"(...) [D]er Legalitätsglaube ist, eingelebt', also selbst traditionsbedingt: Sprengung der Tradition vermag ihn zu vernichten. Und er ist auch charismatisch in dem negativen Sinn: dass hartnäckige eklatante Misserfolge jeder Regierung zum Verderben gereichen, ihr Prestige brechen und die Zeit für charismatische Revolutionen reifen lassen."154

Dieser "sekundäre Traditionalismus"155 ist allerdings nur dann nicht ganz unverständlich, ${ }^{156}$ wenn man - entgegen Weber - annimmt, dass die in Staat und Gesellschaft ausgeübte legale Herrschaft ${ }^{157}$ ihre Qualität dem Modus verdankt, mit dem das erzeugte Recht gewusst wird. Trotz

und anfänglich durch die Autorität einer priesterlichen Rechtsoffenbarung abgestützt (ebd., 449).

${ }^{150}$ Siehe WEBER, Wirtschaft und Gesellschaft (Fn. 134), 448.

${ }^{151}$ Siehe WEBER, Wirtschaft und Gesellschaft (Fn. 134), 456-467.

152 Siehe dazu bei SCHLUCHTER, Die Entwicklung des okzidentalen Rationalismus (Fn. 143), 131. Von der willkürlichen Setzung ist das spezifisch juristische Räsonnement zu unterscheiden. Siehe WEBER, Wirtschaft und Gesellschaft (Fn. 134), 512.

153 WEBER, Wirtschaft und Gesellschaft (Fn. 134), 20.

${ }^{154}$ Ebd., 154. Siehe auch DERS., Staatssoziologie (Fn. 143), 107: „Der Bestand auch bei weitem der meisten, ihrem Grundcharakter nach legalen Herrschaftsverhältnisse ruht, soweit bei ihrer Stabilität der Legitimitätsglauben mitspricht, auf gemischten Grundlagen. Traditionale Eingewöhnung und ,Prestige' (Charisma) rücken mit dem - letztlich ebenfalls eingelebten - Glauben an die Bedeutung der formalen Legalität zusammen: die Erschütterung eines von ihnen durch gegenüber der Tradition ungewohnte Anforderungen an die Beherrschten, außergewöhnliches, das Prestige vernichtende Missgeschick oder Verletzung der üblichen formalen legalen Korrektheit erschüttert den Legitimitätsglauben in gleichem Maße."

155 So HABERMAS, Theorie des kommunikativen Handelns, Bd. 1 (Fn. 23), 361.

156 Ansonsten wäre schwer verständlich, weshalb die legale Herrschaft neben der traditionalen und charismatischen einen eigenständigen Typus bilden kann. Auf dieses Problem weist auch HABERMAS, Theorie des kommunikativen Handelns, Bd. 1 (Fn. 23), 360, hin.

157 Siehe WEBER, Staatssoziologie (Fn. 143), 100. 
der beliebigen Setzbarkeit des Rechts ${ }^{158}$ borgt sich die legale Herrschaft durch die Modalität, um das erzeugte Recht zu wissen, von der traditionalen Legitimität das Moment von Unverfügbarkeit in der Form der Gegenständlichkeit der generell abstrakten Norm ab. ${ }^{159}$ Sie ist vorgeblich das Objekt einer "logischen Sinndeutung", die in der Bildung "fester Rechtsbegriffe" ihren Abschluss findet. ${ }^{160}$ Von der charismatischen Legitimität übernimmt sie ebenfalls im Medium rechtlichen Wissens die Verankerung in einem Berufsstand, der eine standardisierte Verfahrensweise kultiviert. ${ }^{161}$ In der entzauberten Welt wird die Rationalität selbst zum Bann:162

„Der Fortschritt der gesellschaftlichen Differenzierung und Rationalisierung bedeutet also, wenn auch nicht absolut immer, so im Resultat durchaus normalerweise, ein im ganzen immer weiteres Distanzieren der durch die rationalen Techniken und Ordnungen praktisch Betroffenen von deren rationaler Basis, die ihnen, im ganzen, verborgener zu sein pflegt als dem, Wilden' der Sinn der magischen Prozeduren seines Zauberers." 163

Die juristische Expertise, in welcher dem Laien erklärt wird, was gilt, ist für diesen ebenso ein Orakel wie der Schuldspruch der Geschworenen für den Fachjuristen. ${ }^{164}$ Die Reichweite dieser magischen Verhältnisse entgeht Weber nicht gänzlich, wenn er zu denjenigen, die ihrem Zauber erliegen, auch jene zählt, die formale Prozeduren durchführen. 165

158 Siehe WEBER, Wirtschaft und Gesellschaft (Fn. 134), 125; DERS., Staatssoziologie (Fn. 143), 99.

159 Siehe hiezu auch GEORG LUKÁCS, Geschichte und Klassenbewußtsein (1922). 10. Aufl., Neuwied 1988, 189.

160 Siehe WEBER, Wirtschaft und Gesellschaft (Fn. 134), 396.

161 Siehe WEBER, Staatssoziologie (Fn. 143), 108. „Mit der Formalisierung der Vernunft wird Theorie selbst, soweit sie mehr als ein Zeichen für neutrale Verfahrensweisen sein will, zum unverständlichen Begriff (...)." Siehe MAX HORKHEIMER/THEODOR W. ADORNO, Dialektik der Aufklärung. Philosophische Fragmente (1947). Frankfurt am Main 1969, 85. Siehe auch MAX HORKHEIMER, Zur Kritik der instrumentellen Vernunft. Frankfurt am Main 1967, 30-33.

162 Siehe zum Zusammenhang mit Horkheimer und Adorno hier nur HABERMAS, Theorie des kommunikativen Handelns (Fn. 23), 474. Zum Bann, den die jüdisch-christliche Weltanschauung über das Abendland verhängt hat, siehe nun auch MÜNCH, Dialektik der Kommunikationsgesellschaft (Fn. 37), 71-72.

163 MaX Weber, Methodologische Schriften. Herausgegeben von JoHAnNes WinCKELMANN. Frankfurt am Main 1968, 212-213.

164 Siehe zu letzterem WEBER, Wirtschaft und Gesellschaft (Fn. 134), 511.

165 WEBER, Wirtschaft und Gesellschaft (Fn. 134), 512. 


\section{Elliptische Generalisierung}

Die elliptische Generalisierung des juristischen Gutachtens beruht darauf, die im doppelt konvertierten Medium zwischen Deckung und generalisiertem Wert klaffende Lücke (die sich in der Differenz zwischen der Selbstreflexion des Machtmediums und der idealen Selbstreflexion rechtlichen Wissens manifestiert) durch die Unterstellung der Faktizität juristischer Diskurse zu schließen. Diese Faktizität wird als unhintergehbar erlebt. Als soziales Faktum erscheint rechtliches Wissen demnach gerechtfertigt, weil es immer schon ein soziales Faktum ist und nichts außerdem. Der von den Medien, die an die juristische Expertise gekoppelt sind, weitergegebene Verweis auf eine Fundierung, die jenseits des Verwertungskontexts liegt, wird auf diesen selbst zurückgebogen. Die Selbstreflexion wird dementsprechend tautologisch. Aussagen, in denen behauptet wird, was das Recht ist, tragen den Index vorhandener Praktiken der Problemlösung, in denen sich eine "Wertsubstanz" realisiert, die nur die gespenstischen Züge jenes rechtlichen Wissens trägt, das sich als Rechtserkenntnis legitimiert sehen will. ${ }^{166}$ Festmachen lässt sich diese Wertsubstanz auch bloß an den argumentativen Möglichkeiten, die juristischen Kommunikationen zur Verfügung stehen. ${ }^{167} \mathrm{Im}$ Rahmen dieser zirkulären, eines zentralen Brennpunktes entratenden "Selbstreferenz" wird die Begründungsambition ersetzt durch die kontextspezifische Institutionalisierung eines simulierten Traditionalismus. In Anlehnung an Luhmann kann man sagen, dass er sich an der zentralen Bedeutung von "Redundanz" kenntlich macht. ${ }^{168}$ Die juristische Expertise darf nichts Überraschendes kommunizieren.

Die Substanz der juristischen Expertise wird solcherart von ihrer Erscheinungsform in Besitz genommen. ${ }^{169}$ Denn während die symbolische Generalisierung wenigstens noch in Aussicht stellt, dass sich eine Grundlage rechtlichen Wissens reflexiv aufweisen ließe, basiert die elliptische Generalisierung auf der Abstraktion von Begründungen, die

166 Siehe für die abstrakte Arbeit in diesem Sinne KARL MARX, Das Kapital. Kritik der politischen Ökonomie. Bd. 1, MEW Bd. 23, Berlin 1973, 52; GEORG LOHMANN, Indifferenz und Gesellschaft. Eine kritische Auseinandersetzung mit Marx. Frankfurt am Main 1991, $160-163,184,316-323$.

167 In diesen Sinne korrekt NIKLAS LUHMANN, Die Codierung des Rechtssystems, in: Rechtstheorie 17 (1986), 171-203.

168 Siehe Alicia JUARRERO-ROQUÉ, Fail-Safe Versus Safe-Fail: Suggestions Toward an Evolutionary Model of Justice, in: Texas Law Review 69 (1991), 1745-1778, hier: 17641768 .

169 Siehe zu einer ähnlichen Beobachtung hinsichtlich der Äquivalenzbestimmtheit der Waren LOHMANN, Indifferenz und Gesellschaft (Fn. 166), 208-209. 
über den Verwertungskontext juristischer Gutachten hinausverweisen. Daher kann einer Aussage die metasprachliche Eigenschaft, das geltende Recht darzustellen, nur zukommen, wenn sie sich einer Sprachpraxis zuordnet (sich in ihr ",ausdrückt"170), in welcher die methodische Selbstreflexion eine Schlacke an unverbundenen Argumentations- und Verfahrensweisen zurückgelassen hat, die dazu verhelfen, eine juristische Expertise zu artikulieren. ${ }^{171}$ Dieser Fundus - das "Medium" - steht für die sachliche Auflösungskraft juristischer Argumente, über deren jeweilige Zusammensetzung kontextgebundene Überzeugungen den Ausschlag geben. ${ }^{172}$

Die Redundanz der juristischen Expertise - das graue Einerlei ihrer immergleichen Struktur und ihrer schematischen Argumente - ist es, welche das Zusammenspiel von Deckung und generalisiertem Wert simuliert. An die Stelle der Selbstreflexion der Begründung tritt die Teilhabe der juristischen Diskurse am generalisierten Medium (dem "Sprachspiel"). ${ }^{173}$ Bei Luhmann wird dies folgendermaßen zum Ausdruck gebracht:

"Es gibt keinen anderen ,Grund' für Erkenntnisse [als eine bereits in Gang gekommene Praxis, A.S.]. (...) [J]edenfalls kann ein Beobachter nur ,Gründe' sehen, die das System wählt, um begründen zu können. So gesehen begründet das Begründen den Grund - und nicht umgekehrt. Das Interesse des Beobachters (der auch das Wissenschaftssystem selbst sein kann) richtet sich damit auf die Frage, wie man einem grundlosen Grund Feststellungen abgewinnen kann, die im System als richtige Feststellungen praktiziert werden können."174

Redundanz, und dies hat sie nicht zufällig mit der ominösen "différance" gemein, gestattet es somit, Bedingungen der Unmöglichkeit in Bedingungen der Möglichkeit zu verwandeln. ${ }^{175}$ Der Argumentierende

170 Siehe zum Verhältnis von einfacher und relativer Wertform bei MARX, Das Kapital I (Fn. 166), 64-69, 70-74; dazu LOHMANN, Indifferenz und Gesellschaft (Fn. 166), 190$191,199$.

${ }^{171}$ Insofern lässt sich auf elliptische Generalisierung die Unterscheidung von Medium (lose Koppelung von Elementen) und Form (rigide Koppelung) anwenden. Siehe dazu LUHMANN, Die Wissenschaft der Gesellschaft (Fn. 20), 53, 182-185; DIRK BAECKER, Einleitung, in: DERS. (Hrsg.), Probleme der Form. Frankfurt am Main 1993, 9-21, hier: 20.

172 Siehe Analoges zur Wahrheit als dem Medium der Wissenschaft bei LuHMANN, Die Wissenschaft der Gesellschaft (Fn. 20), 185.

173 Auch dieser Aspekt wird richtig erkannt bei NikLas LuHManN, Die Einheit des Rechtssystems, in: Rechtstheorie 14 (1983), 129-154.

${ }^{174}$ LuHMANN, Die Wissenschaft der Gesellschaft (Fn. 20), 390-391.

175 Siehe JACQUES DERRIDA, Grammatologie. Frankfurt am Main 1974, 248. 
kann in ein Spiel eintreten, "(...) in dem, wer verliert, gewinnt, und in dem man mit jedem Zug gewinnt und verliert."176

Das seinen Verwertungskontext bezeichnende rechtliche Wissen substituiert das leerlaufende "theoretische" Begründen durch die Auszeichnung eines "Fachdiskurses", an dem die im Plural auftretenden Praktiken der Rechtsauslegung partizipieren. ${ }^{177}$ Das Recht nimmt sich als ",autopoietisches System" aus. ${ }^{178}$ Allerdings ist der Selbstbezug eines rechtlichen Wissens, das als System von Kommunikationen erscheint, eine Folge von interner Vermachtung. Die letztere gibt darüber den Ausschlag, dass sich in den Augen des Beobachters die gelingende Rechtserkenntnis nicht mehr von der Ausübung institutioneller Autorität unterscheiden lässt. ${ }^{179}$

Der rechtstheoretischen Kritik ist es vergönnt, Manifestationen dieses Sachverhalts aufzudecken. ${ }^{180}$ Allerdings kommt ihr in der institutionellen Rechtsverwirklichung kein Ort zu. Die juristische Selbstreflexion wird zum Emblem der Ohnmacht des Rechts. Sie findet, wie in diesem Text, in der Systemumwelt des Rechtssystems statt.

176 JACQUES DERRIDA, Die différance, in: DERS., Randgänge der Philosophie. Frankfurt am Main 1976, 6-38, hier: 28.

177 Siehe zu einem analogen "Springpunkt der Verdinglichung" bei MARX, Kapital I (Fn. 166), 70.

178 Siehe bloß GUNTHER TEUBNER, Recht als autopoietisches System. Frankfurt am Main 1989; NIKLAS LuHManN, Das Recht der Gesellschaft. Frankfurt am Main 1993.

179 Siehe STANLEY FISH, Is There A Text In This Class? The Authority of Interpretive Communities. Cambridge (Mass.) 1980, 14.

${ }^{180}$ Siehe dazu NIKOLAUS FORGÓ, Gewalt ist, was Gewalt ist, in: Juridicum 2/94 (1994), 35-39. 